Chair for Management Sciences and Energy Economics University of Duisburg-Essen

EWL Working Paper No. 03/12

\title{
Technical Uncertainty and Value of Information with Application to Optimal Network Component Replacement
}

by

Stephan Schaeffler, Dominik Schober and Christoph Weber

June 01, 2012 


\title{
Technical Uncertainty and Value of Information with Application to Optimal Network Component Replacement
}

\author{
Stephan Schaeffler, Dominik Schober and Christoph Weber
}

\begin{abstract}
Optimal age replacement policies for network components such as cables, overhead lines or transformers are usually identified based on gathered knowledge about the state of a component and its stochastic deterioration process. In this context, uncertainty is an important challenge because current information about the aging process may be false. Especially in the context of innovative use of newly developed network equipment some experience knowledge from similar equipment might exist or pre-testing under laboratory conditions could allow setting up hypothesis about the characteristics of aging. Nonetheless, substantial uncertainty is still common in replacement. An example in this context is the lifetime of PE tubes in gas networks, which is not very well explored due to the fact that no tubes older than 40 years exist. The length of the aging process as well as the expected starting date can be inferred only to some confidence probability. Apart from newly developed equipment, production imperfections like the Water treeing Effect in cable insulations led to very early replacement of complete lots of cables because the insulation deteriorated much earlier than initially expected.

Hence, the question arises how these different sources of uncertainty will impact the network operator's replacement decision. Further it is of interest how much value can be attributed to the reduction of the uncertainty. In this paper, an optimal replacement strategy in an analytical stationary state model is derived explicitly with local and global optima. Based on a discrete mixture model of failure rates under perfect replacement, we show how different assumptions about the underlying type of uncertainty will affect the replacement decision. In a further step, the value of information representing the cost difference between a state of parameter certainty and the state of parameter uncertainty is derived. Trough the course of some applications, it is shown that the value of information increases with the level of uncertainty. Some exemplary calculations are presented to show that the magnitude of the value of information is significant.
\end{abstract}

Keywords: Replacement decision, uncertainty, age replacement policy, failure rate, value of information

JEL-Classification: C44, D21

STEPHAN SCHAEFFLER

Horváth \& Partners Management Consultants

(Ganghoferstr. 39, 80339 München)

++49 - (0)89 / 544625-15

www.horvath-partners.de

sschaeffler@horvath-partners.com

\section{DOMINIK SCHOBER}

Chair for Management Science and Energy Economics, University of Duisburg-Essen

(Universitätsstr. 11, 45117 Essen)

++49 - (0)2 01 / 183-2903

www.ewl.wiwi.uni-due.de

DOMINIK SCHOBER

Chair for Management Science and Energy Economics,

University of Duisburg-Essen

(Universitätsstr. 11, 45117 Essen)

++49 - (0)2 01 / 183-2903

www.ewl.wiwi.uni-due.de

The authors are sorely responsible for the contents which do not necessarily represent the opinion of the Chair for Management and Energy Economics. 


\section{Introduction}

The introduction of incentive and quality regulation in important network branches such as telecommunication or utilities has led many network operators to reduce maintenance costs by optimizing the trade-off between planned maintenance costs and cost for random breakdowns which may lead to outages and penalties by regulation authorities. Numerous models have been developed within the Operations Research literature, which support network operators in identifying optimal replacement strategies. For an overview, cf. e.g. Valdez-Flores and Feldman (1989) and Wang (2002). However, the task is made much more difficult by a significant level of uncertainty concerning the methodology and data used for these models. Replacement models are founded on assumptions about the current state of the asset and its further deterioration. These assumptions may be wrong. Some important sources of uncertainty are the shape of the distribution function, and the distribution function being known, parameters such as the starting point of the aging process and its speed. The introduction of completely new components or new technologies will lead to a situation, where only rough assumptions may be available. Another situation arises when lots of a good are of very different quality, due to flaws during the production process. In this case, an operator will probably know the aging characteristics of a good and a bad asset. However, he will not be able to know early if he got a good or a bad lot. Such uncertainty is common in replacement. An example is the lifetime of PE tubes where the start and the magnitude of the aging process are rather unclear due to the fact that no tubes older than 40 years exist. Another example is water treeing, a phenomenon regarding cable insulations that led to very early replacement of complete lots of cables because the insulation of a high number of lots deteriorated much earlier than initially expected. Hence, the question arises how such uncertainty will affect the network operator's replacement decision and how to value the information about the correct state of the equipment and the probability that it will fail in the close future. Research so far has mainly focused on different types of maintenance strategies (age replacement, block replacement, repair limits, etc.). Furthermore, distributions that shall reflect deterioration of assets and forecast breakdown probabilities as well as methods to estimate distribution parameters have been discussed. To our knowledge, there is no research which explicitly analyses how uncertainty may affect an agent's replacement strategy in an analytical modeling framework. This may be due to the fact that common failure distributions do not allow an analytical derivation of optimal replacement strategies because of terms, which even do not have closed form representations in many cases.

The focus of the present paper yet lies on the effect of uncertainty on an optimal replacement strategy and its implications for the value of information. In order to derive general insights, a stationary analytical framework is chosen, which models the cost annuity as a sum of planned replacement and breakdown. We will use a uniform unconditional failure distribution which permits to analytically derive an optimal replacement strategy and discuss how uncertainty about the starting point and the speed of aging will affect the optimal replacement strategy. We find that uncertainty will in the majority of cases lead to later replacement.

In the applications section, two different cases will be discussed. If components from suppliers are of different quality and this quality is observable through testing, the value of information corresponds to the willingness to pay for quality control. In application of the approach to the phenomenon of water treeing of the insulation of VPE cables, the value of information is found to be significant. In a second application, a new technology is introduced that slows down deterioration, e.g. a new type of insulation of gas steel tubes. However, its effectiveness is not certain. The impact of uncertainty on the expected cost advantage is derived.

The structure of the paper is as follows: The existing literature is reviewed in section 2. Then a general model of replacement is sketched in section 3. In section 4, the concept of parameter uncertainty will be included in the model and the existence of optimal replacement strategies under uncertainty will be shown. In section 5 we will discuss two exemplary applications using the results obtained so far. A final chapter concludes and provides an outlook for future research. 


\section{Literature Overview}

Replacement models can be characterized as either deterministic or probabilistic. Deterministic replacement models assume a deterioration of the functionality and thus of the output of equipment, but they do not address full breakdown. Probabilistic models focus on the possibility of failure. Uncertainty may result because the distribution function is not known or the parameters of distribution functions (either failure rate or restoration process) are uncertain.

Fox (1966) investigates the effect of uncertainty in the case of a preventive periodic maintenance policy. Uncertainty occurs because the failure distribution function is not known. In this case, the optimal strategy consists of solely replacing at failure and to conduct no planned maintenance at all. As opposed to this extreme uncertainty leading exclusively to corner solutions, usually a decision taking entity has some prior beliefs about aging of assets, which in turn lead to a convex optimization trading off higher costs of shorter actual lifetimes due to premature replacement against higher costs resulting from failure. How a firm will use this knowledge about failure distributions has yet hardly been treated.

A major discussion on how to deal with uncertain parameter focused the restoration process of failed equipment. If one assumes that repair of a good does not lead to a state "as good as new" (i.e. minimal repair that does not reset the time $t$ since the last repair to 0 ), the probability of failure after repair will be more or less close to the probability before repair. In the specific case of Barlow and Hunter (1960), the probability of failure in a periodic PM policy is governed by a Poisson process, whose parameters are assumed to be known. Introducing uncertainty in the restoration process can be achieved by different specifications for the Poisson process, which all have based on different observations. Bassin (1973) uses a Bayesian estimation approach to deduce the parameters for the Poisson process and thus derives optimal periodic PM in the case of minimal repair. Dogramaci and Fraiman (2004) use the basic optimal control model by Kamien and Schwatz (1971) in a dynamic setting with multiple periods and introduce uncertainty (caused by technological change) by considering alternative scenarios using this base model. Therefore, they account for the fact that future technologies and their maintenance requirements are supposed to be unknown. They numerically deduce the optimal strategy for each scenario, their focus lying on the minimization of computation times and the effect of technological jumps on maintenance costs. Wang et al. (2009) present a condition-based replacement model for a deteriorating system with a number of identical assets. In their model, deterioration of an asset increases with time but whether a failure occurs or not depends on the condition-based reliability adding a layer of uncertainty.

Parameter uncertainty regarding the distribution of the failure rate is another important topic. Sathe and Hancock (1972) present a Bayesian model for determining optimal maintenance policies when no historical data about failure rates are given. The decision makers' beliefs (which are represented by probability density functions regarding the relevant parameters) are used as prior for the distribution parameter. Based on more and more observations, these beliefs are recursively updated using Bayes' theorem. Mazzuchi and Soyer (1996) present a comparable approach based on Weibull failure distributions with unknown coefficients. Uncertainty is reassessed as all data gathered during the process is used to increase the estimation quality of the distribution parameters and consequently replacement strategies are adapted. Juang and Anderson (2004) extend this approach by uncertain repair costs and by a more complete set of maintenance actions such as minimal repair, major repair, planned replacement, unplanned replacement and periodic scheduled maintenance. Finkelstein (2008) points out an interesting aspect we will refer to later. If one is not sure about the parameters of the distribution function, he can use another distribution function regarding these parameters. However, conventional approaches to find optimal replacement strategies require specific properties of the failure rate function. Barlow and Hunter (1960) find that, given an age replacement protocol, a general optimum only occurs if the conditional failure rate is strictly non-decreasing (IFR-condition). Typical failure distributions such as the exponential distribution, the gamma distribution, the truncated normal distribution and the Weibull distribution fulfill this IFR condition. Finkelstein (2008) states that a combination of failure rates (e.g. Weibull distribution and Gamma distribution) may lead to a distribution which does not fulfill the IFR-condition. Hence uncertainty about the distribution parameters may lead to a situation where the derivation of optimal replacement strategies is not trivial, because 
multiple local optima exist. A branch of literature discussing a problem relatively close to network replacement under uncertainty focuses on technological change and its impact on optimal service life. Yatsenko and Hritenko (2008) for instance discuss the properties of optimal replacement under technological change in a dynamic replacement setting. Based on an amount of literature they summarize, they claim that "qualitative properties of optimal machine replacement under technological change are poorly known".

The value of information has not been a predominant topic in maintenance and replacement literature so far. In the context of supply chains and stock policy, the value of information is the result of the degree of sharing information between supplier, producer and retailer. Huang et al. (2003) review more than 100 contributions about the impact of sharing production information on supply chain dynamics. Lee (2008) presents a Bayesian approach to determine the value on information if ordered quantities cannot be carried forward to future periods. Ketzenberg (2009) discusses uncertainty regarding demand, recovery yield and capacity utilization in the context of closed loop supply chains. His study indicates which type of information is most valuable for different conditions.

Summarizing, we state that many authors primarily focus on methods of estimation to reduce uncertainty; very few analyze effects of uncertainty itself. In particular the effect of discrete uncertainty, where the distribution ex ante is a mixture of two alternative distributions, is hardly treated. However this case is of considerably practical importance, both in the case of lots of diverging quality (as in the water treeing case) and when it comes to estimate the value of information obtained from ex-ante quality control. By focusing on an analytically tractable case, moreover it is also possible to derive general insights which are valid beyond the particular numerical examples considered.

\section{General Age Replacement Model}

In the following section, the stochastic replacement model is presented briefly. ${ }^{1}$ Consider a network operator that optimizes his age replacement strategies. The costs considered correspond to the capital expenditure for a planned replacement of equipment $Z$ and additional costs for breakdowns $S^{2}$. The decision variables are the replacement ages $t_{j}$ at which asset $j$ is subject to planned replacement. In order to keep the problem analytically tractable, we chose a stationary setting and focus on the equilibrium state. Thus, time in our model is represented by the index $t$ (which can be considered equivalent to the asset age) and the replacement age $t_{j}{ }^{3}$ The objective function is:

$$
\min _{t_{j}} C\left(t_{j}\right)=\min \left(Z\left(t_{j}\right)+S\left(t_{j}\right)\right)
$$

The link between unconditional failure rate $f(t)$ and conditional failure rate $r(t)$, is given by:

$$
r(t)=\frac{f(t)}{1-F(t)}
$$

Another expression necessary to identify the optimal replacement age in a stationary state is given by the expected utilization period $G\left(t_{j}\right)$ given the replacement age $t_{j}$. It corresponds to:

$$
G\left(t_{j}\right)=\int_{o}^{t_{j}}(1-F(t)) d t
$$

$G\left(t_{j}\right)$ and the cumulative survival distribution $1-F(t)$ are required to identify the vintage distribution $H\left(t, t_{j}\right)$ that will occur in stationary state:

\footnotetext{
${ }^{1}$ See Weber et al. (2010) for a more detailed description of the model.

${ }^{2}$ This may also include quality penalties in e.g. regulated infrastructure industries.

${ }^{3}$ In the context of this analytical model, the initial (or starting) age of an asset is not of relevance. In dynamic models however, the initial age structure of equipment is highly relevant in the context of optimization.
} 


$$
H\left(t, t_{j}\right)=\frac{1-F(t)}{G\left(t_{j}\right)}
$$

Equipment that fails has to be replaced, which leads to the differentiation of preventive replacement $R_{\text {prev }}$ (due to the fact that the asset's age attains the decision variable replacement age, thus $t=t_{j}$ ) and corrective replacement $R_{\text {corr }}$ (due to failure while $t<t_{j}$ ). Corrective replacement is given by:

$$
R_{\text {corr }}\left(t_{j}\right)=\int_{0}^{t_{j}}\left(H\left(t, t_{j}\right) \cdot r(t)\right) d t=\int_{0}^{t_{j}}\left(\frac{1}{G\left(t_{j}\right)} \cdot f(t)\right) d t=\frac{F\left(t_{j}\right)}{G\left(t_{j}\right)}
$$

Planned replacement can be calculated as follows:

$$
R_{\text {prev }}\left(t_{j}\right)=H\left(t_{j}, t_{j}\right)=\frac{1-F\left(t_{j}\right)}{G\left(t_{j}\right)}
$$

With $K$ corresponding to the unit costs for replacement, the total replacement costs add up to:

$$
Z\left(t_{j}\right)=K \cdot\left(R_{\text {prev }}\left(t_{j}\right)+R_{\text {corr }}\left(t_{j}\right)\right)=\frac{K}{G\left(t_{j}\right)}
$$

For each failure, additional cost of $s$ result. We can write:

$$
S\left(t_{j}\right)=s R_{\text {prem }}=\frac{s F\left(t_{j}\right)}{G\left(t_{j}\right)}
$$

The total cost amount to:

$$
C\left(t_{j}\right)=\frac{K+s F\left(t_{j}\right)}{G\left(t_{j}\right)}
$$

with the resulting optimality condition (OC) for an inner optimum:

$$
\begin{aligned}
& \frac{\partial C\left(t_{j}\right)}{\partial t_{j}}=\frac{\partial Z\left(t_{j}\right)}{\partial t_{j}}+\frac{\partial S\left(t_{j}\right)}{\partial t_{j}}=0 \\
& \Leftrightarrow \frac{s f\left(t_{j}\right) G\left(t_{j}\right)-\left(K+s F\left(t_{j}\right)\right)\left(1-F\left(t_{j}\right)\right)}{G\left(t_{j}\right)^{2}}=0
\end{aligned}
$$

Writing $K_{s}=K / s$ and using equation (2) the OC thus can be transformed to:

$$
r\left(t_{j}\right) G\left(t_{j}\right)-F\left(t_{j}\right)-K_{s}=0
$$

In order to derive analytical insights concerning the effect of uncertainty in the decision process, one has to make assumptions about the functions describing the wear-out effect. The functions most commonly used to model lifetime assumptions are the Normal distribution, the Weibull distribution and the Exponential distribution.4 In order to keep the problem analytically tractable, we use a uniform distribution for $f$ (cf. Figure 1). An asset shows no sign of aging at all until $t_{a}$, then it deteriorates between $t_{a}$ and the point $t_{b}$, where all assets have failed. The use of a linear distribution function is a valid method to approximate frequently used lifetime distribution functions.

\footnotetext{
${ }^{4}$ For an overview over commonly used functions and their properties, cf. Finkelstein (2008).

${ }^{5}$ In the Appendix A to this article, we show how a linear distribution may be used to approximate a Weibull distribution and how such an approximation has relatively low impact on the optimal replacement age. Approximation quality may even be enhanced by using multiple uniform distributions sum up to a piecewise definition of the cumulative lifetime distribution. The appendix is available online or upon request.
} 


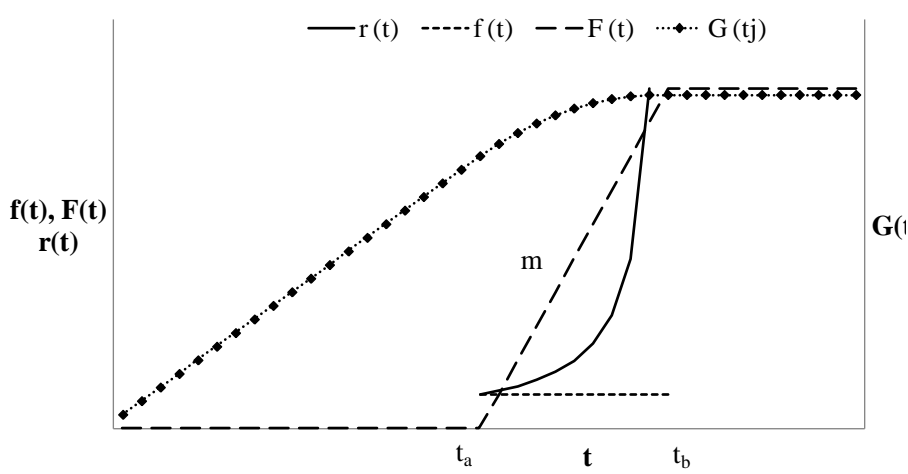

Figure 1: Uniform distribution of $f$

One of its major advantages is that optimal replacement strategies can be derived analytically. ${ }^{6}$ The conditional failure rate is given by:

$$
r(t)=\left\{\begin{array}{ll}
0 & \text { for } t<t_{a} \text { or } t_{b}<t \\
\frac{m}{1-\left(m\left(t-t_{a}\right)\right)} & \text { otherwise }
\end{array}\right\}
$$

The unconditional failure rate corresponds to:

$$
f(t)=\left\{\begin{array}{ll}
0 & \text { for } t<t_{a} \text { or } t_{b}<t \\
m & \text { otherwise. }
\end{array}\right\}
$$

The cumulative failure distribution is:

$$
F(t)=\left\{\begin{array}{ll}
0 & \text { for } t<t_{a} \\
m\left(t-t_{a}\right) & \text { for } t_{a} \leq t<t_{b} \\
1 & \text { for } t \geq t_{b}
\end{array}\right\}
$$

And finally, the expected lifetime corresponds to:

$$
G\left(t_{j}\right)=\left\{\begin{array}{ll}
t_{j} & , \text { for } t_{j}<t_{a} \\
t_{j}-\frac{m}{2}\left(t_{j}-t_{a}\right)^{2} & , \text { for } t_{a} \leq t_{j}<t_{b} \\
t_{b}-\frac{m}{2}\left(t_{b}-t_{a}\right)^{2} & , \text { for } t_{j} \geq t_{b}
\end{array}\right\}
$$

Based on (10), the optimal replacement strategy can be derived, it corresponds to: ${ }^{7}$

$$
t_{j}^{*}=t_{a}+\frac{\sqrt{K_{s}^{2}+2 K_{s}-2 m t_{a}}-K_{s}}{m}
$$

One can see, that a steeper slope $m$, lower cost for planned replacement $K$, higher penalty cost $s$ and an earlier beginning of the aging process $t_{a}$ will lead to earlier replacement. Inserting the optimal replacement strategy in the cost function, one obtains:

\footnotetext{
${ }^{6}$ Regarding the previously mentioned distribution laws, such an approach is not possible, cf. Barlow and Proschan (1964).

${ }^{7}$ Cf. Weber et al. (2010).
} 


$$
C=s \frac{\sqrt{K_{s}^{2}+2 K_{s}-2 m t_{a}}}{t_{a}+\frac{1}{2 m}-\frac{1}{2 m}\left(\sqrt{K_{s}^{2}+2 K_{s}-2 m t_{a}}-K_{s}-1\right)^{2}}
$$

Based on (16), the impact of parameters on overall stationary replacement cost can be deduced. An increase of planned replacement cost $K$ implies an increase of $K_{s}$ resulting in an increase of the numerator and a decrease of the denominator. Consequently, the total cost increases with $K$. However, the impact of a higher $s$ is more difficult to assess with the first term increasing and the denominator decreasing (which is due to the effect, that a higher $s$ will reduce the replacement interval. By formally deriving, we find that the total cost increases with additional breakdown costs. A later start of the aging process (represented by a higher $t_{a}$ ) will reduce annual replacement cost as it decreases the numerator and increases the denominator. A more rapid deterioration, which will lead to a higher $m$, has the opposite effect as it will lead to earlier replacement. The nature of the replacement model is stochastic, as failures occur with a conditional failure rate $r(t)$. Nevertheless, the parameters of the distribution function are assumed to be certain. This means that if a network operator holds an infinite number of assets, the costs will converge against the expectation. The assumption of parameter certainty is commonly encountered in replacement literature but will be loosened subsequently.

\section{Model of Age Replacement under Distribution Parameter Uncertainty}

Often, especially if a new technology is introduced, distribution functions will not be known with certainty but will be built upon uncertain expectations. If expectations are wrong, which has for instance happened in the case of Water treeing cables, suboptimal replacement strategies will be implemented and additional costs result. To discuss the effect of parameter uncertainty, $m$ and $t_{a}$ are now considered as uncertain parameters. ${ }^{8}$ The uncertainty is modeled as discrete mixtures of two possible failure distributions (e.g. bad vs. good components) with probabilities $\vartheta$ (for the unfavorable failure function) and $1-\vartheta$. In the case of uncertainty about $t_{a}$, parameter uncertainty is represented by a deviation $c$ from the average start time $t_{a}$ and end time $t_{b}$ of the aging period. In the case of uncertainty about $m$, parameter uncertainty is characterized through an additive or subtractive term $z$ modifying $m$. Figure 2 exemplarily presents the cumulative failure distributions for these two cases. In the left part, two failure distributions with distinct starting age and overlapping aging periods are shown. In the right part, two cumulative failure distributions starting both in $t_{a}$, one being slow, one being fast, are mixed to form the distribution denominated 'uncertain'.
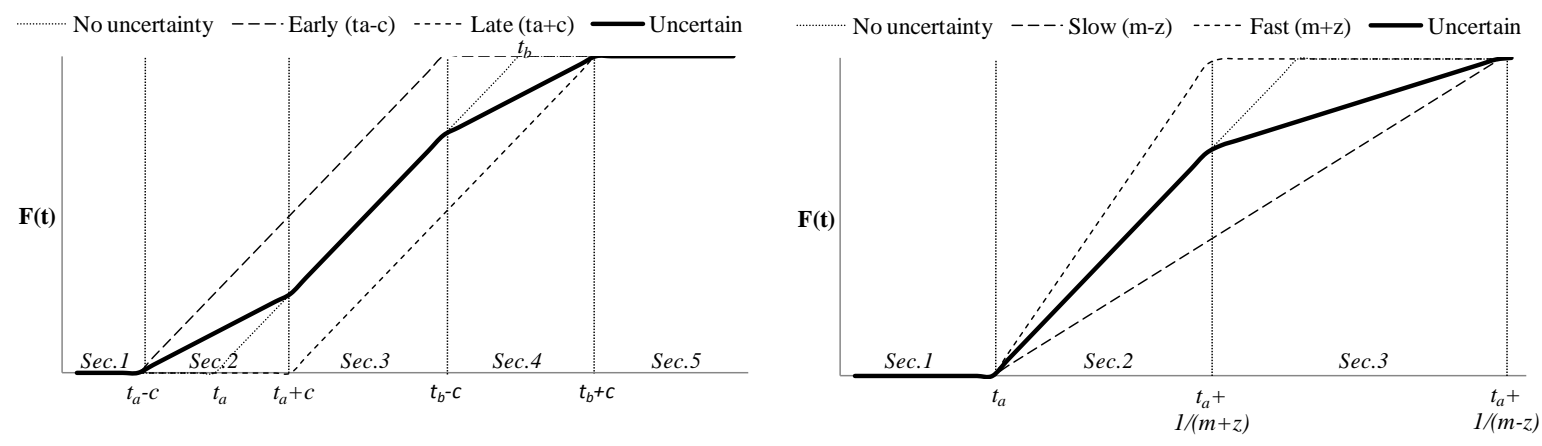

Figure 2: Uncertainty about start of aging (left hand) and slope (right hand)

A discrete mixing of two failure rates may appear limiting at first glance. Yet, one has to bear in mind that decision finding processes in practice are often handled with the use of a relatively low number of scenarios, notably by using a worst case and best case scenario plus the expectation scenario (under assumed parameter certainty). Second, the scenarios may be

\footnotetext{
${ }^{8} t_{b}$ is implicitly defined by the specification of $t_{a}$ and $m$, because $t_{b}=t_{a}+1 / m$.
} 
used to represent expectations of two distributions, a best case and a worst case distribution, and thus leave significant freedom of parameterization. Third, this freedom of parameterization can be used to approximate arbitrary (empirical or simulated) functions. The mixing of a first unfavorable failure rate distribution (indexed $U$, e.g. early or fast aging) with a favorable failure rate distribution (indexed $F$, e.g. late or slow aging) leads to mixed unconditional failure rate $(M)$ :

$$
f_{M}(t)=\vartheta f_{U}(t)+(1-\vartheta) f_{F}(t)
$$

The cumulative failure distribution $F(t)$ is derived analogously. For the expected lifetime, we again obtain:

$$
G_{M}\left(t_{j}\right)=\int_{o}^{t_{j}}\left(1-F_{M}(t)\right) d t
$$

The mixed functions may now be used to update the OC

$$
r_{M}\left(t_{j}\right) G_{M}\left(t_{j}\right)-F_{M}\left(t_{j}\right)-K_{s}=0
$$

The resulting optimal replacement age under parameter uncertainty will be denominated $t_{j}^{* *}$ (in contrast to $t_{j}^{*}$ under parameter certainty). In order to analytically derive this optimal replacement strategy, we now turn to the relevant cases.

\subsection{Optimal replacement when the start of aging is uncertain}

Depending on the range of uncertainty, the intervals during which aging is expected to take place will either overlap or not. These two cases will be referred to as the overlapping and the non-overlapping case. We will discuss the overlapping case with priority and show modifications occurring in the non-overlapping case. Both cumulated failure distributions have the same slope. Further a symmetric range $c$ around the initial starting point $t_{a}$ is defined. In the case of $\mathrm{cm} \leq 0.5$, the two distribution functions will be overlapping, for $\mathrm{cm}>0.5$, they will be non-overlapping.
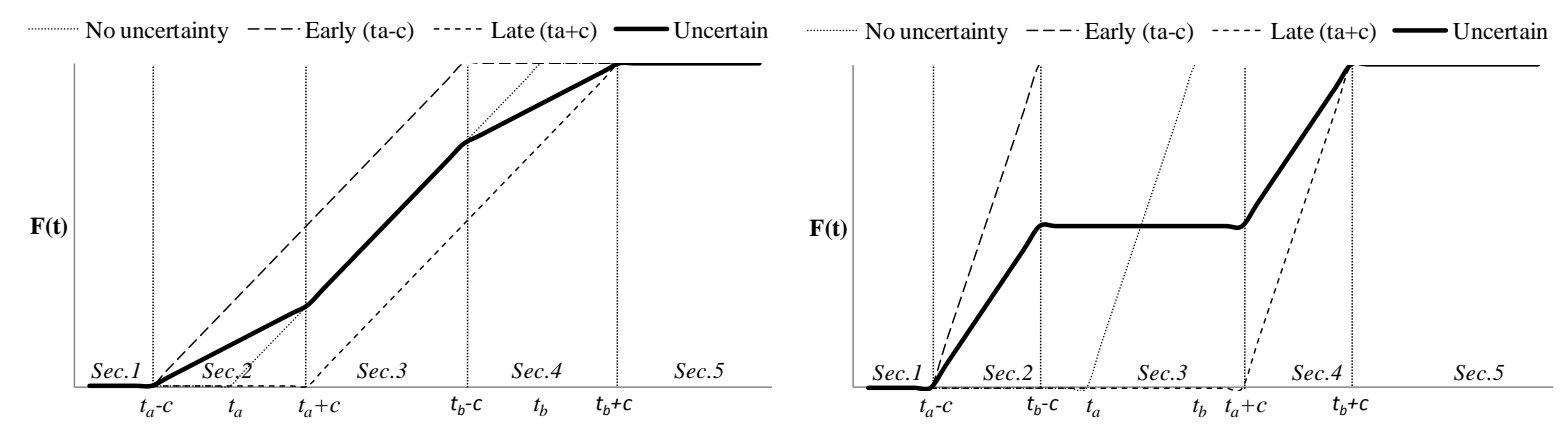

Figure 3: Overlapping (left hand) and non-overlapping case (right hand)

\section{Case A: Overlapping Failure Distributions}

Two states can be differentiated: early aging (i.e. aging starts at $t_{a}-c$ and ends at $t_{b}-c$ ) and late aging (i.e. aging starts at $t_{a}+c$ and ends at $t_{b}+c$ ). In the case of the overlapping specification, the uncertainty implies five different sections for the aggregated failure function. In the first and the fifth section, no aging occurs at all (cf. Figure 3, left hand side). In the second section of the function, aging will only occur under early aging. In the third section, aging will occur in both states, thus the slope of the expected cumulative distribution will also be higher. In the fourth section, aging will only occur under late aging. In the following application, we will refer to the range $\left[t_{a}-c ; t_{a}+c\left[\right.\right.$ as section $2,\left[t_{a}+c ; t_{b}-c[\right.$ as section 3 and to $\left[t_{b}-c ; t_{b}+c\right.$ [ as section 4 . These are the most interesting sections, because optima for the replacement age are only to be expected here. 
Proposition 1 The unconditional failure rate of the mixture distribution corresponds to

$$
f_{M}(t)= \begin{cases}0 & \text { for } t<t_{a}-c \text { or } t>t_{b}+c \\ \vartheta_{m} & \text { for } t_{a}-c \leq t<t_{a}+c \\ m & \text { for } t_{a}+c \leq t<t_{b}-c \\ (1-\vartheta)_{m} & \text { for } t_{b}-c \leq t \leq t_{b}+c\end{cases}
$$

The cumulative failure distribution, i.e. the probability that the equipment will fail and have to be replaced within $t$ is given by:

$$
F_{M}(t)= \begin{cases}0 & \text { for } t<t_{a}-c \\ \vartheta m\left(t-\left(t_{a}-c\right)\right) & \text { for } t_{a}-c \leq t<t_{a}+c \\ \vartheta m\left(t-\left(t_{a}-c\right)\right)+(1-\vartheta) m\left(t-\left(t_{a}+c\right)\right) & \text { for } t_{a}+c \leq t<t_{b}-c \\ \vartheta+(1-\vartheta) m\left(t-\left(t_{a}+c\right)\right) & \text { for } t_{b}-c \leq t<t_{b}+c \\ 1 & \text { for } t_{b}+c \leq t\end{cases}
$$

And the expected age of survival corresponds to:

$$
G_{M}\left(t_{j}\right)= \begin{cases}t_{j} & \text { for } t_{j}<t_{a}-c \\ \vartheta\left(t_{j}-\frac{m}{2}\left(t_{j}-\left(t_{a}-c\right)\right)^{2}\right)+(1-\vartheta) t_{j} & \text { for } t_{a}-c \leq t_{j}<t_{a}+c \\ \vartheta\left(t_{j}-\frac{m}{2}\left(t_{j}-\left(t_{a}-c\right)\right)^{2}\right)+(1-\vartheta)\left(t_{j}-\frac{m}{2}\left(t_{j}-\left(t_{a}+c\right)\right)^{2}\right) & \text { for } t_{a}+c \leq t_{j}<t_{b}-c \\ \vartheta\left(t_{b}-c-\frac{1}{2 m}\right)+(1-\vartheta)\left(t_{j}-\frac{m}{2}\left(t_{j}-\left(t_{a}+c\right)\right)^{2}\right) & \text { for } t_{b}-c \leq t_{j}<t_{b}+c \\ \vartheta\left(t_{b}-c-\frac{1}{2 m}\right)+(1-\vartheta)\left(t_{b}+c-\frac{1}{2 m}\right) & \text { for } t_{b}+c \leq t_{j}\end{cases}
$$

Proofs of Proposition 1 Cf. Appendix B.1.

Given the piecewise definitions, the functions $F_{M}$ and $G_{M}$ are continuous, yet given the piecewise constant $f_{M}$ the optimality condition is not necessarily continuous or even smooth. Therefore, multiple local optima plus corner solutions might be possible. For the identification of the global optimum the following procedure can be followed:

Step 1: The inner local optimum for each section is identified.

Step 2: Characteristics of solutions are discussed.

Step 3: A feasibility analysis is conducted by comparing one specific section's local optimum to the support of the respective section.

Step 4: If multiple local solutions are found, then the global optimum has to be identified by comparison of the cost of the different solutions considering corner solutions as well.

Proposition 2 Using optimality condition (9) the interior optima for the different sections can be derived. The solutions for section 1 and 5 are corner solutions, namely $t_{a}-c$ (section 1) and $t_{b}+c$ (section 5). The inner solution for sections 2, 3 and 4 are given by the following formulas.

Section 2:

$$
t_{j}^{* *}=t_{a}-c+\frac{\sqrt{K_{s}^{2}+2 K_{s}-2 \vartheta m\left(t_{a}-c\right)}-K_{s}}{\vartheta_{m}}
$$


Section 3:

$$
t_{j}^{* *}=t_{a}+\frac{c m(1-2 \vartheta)+\sqrt{K_{s}^{2}+2 K_{s}-2 m t_{a}-2 c m\left((1-2 \vartheta)-2 c m\left(\vartheta-\vartheta^{2}\right)\right)}-K_{s}}{m}
$$

Section 4:

$$
t_{j}^{* *}=t_{a}+c+\frac{\sqrt{K_{s}^{2}+2 K_{s}-2 m t_{a}+\vartheta+2 \vartheta m t_{a}+2 c m\left(3 \vartheta-1-2 \vartheta^{2}\right)}-\left(\vartheta+K_{s}\right)}{(1-\vartheta) m}
$$

\section{Proofs of Proposition 2 Cf. Appendix B.2.}

The main purpose of this article is to investigate how uncertainty affects optimal replacement behavior. The parameters representing uncertainty are, first, $\vartheta$, the probability of occurrence of the early aging case vs. the late aging case, and, second, $c$, the degree of uncertainty, i.e. the distance between early and late aging cumulative failure functions in years. The following table summarizes the results of respective derivations.

Table 1: Dependence of optimal replacement on $\vartheta$ and $c$

\begin{tabular}{lll}
\hline \hline Inner optimum & $\partial \boldsymbol{t}_{j}^{* * *} / \partial \boldsymbol{} \vartheta$ & $\partial \boldsymbol{t}_{j}^{* * *} / \partial \boldsymbol{c}$ \\
\hline \hline Section 2 & $<0$ & $<0$ for $K_{s}>1$ \\
Section 3 & $<0$ & Depends on $\vartheta$ : Typically \\
& $($ sufficient condition: & $<0$ for $\vartheta>0.5$ \\
& $\left.K_{s} \geq 3 / 2\right)$ & $>0$ for $\vartheta \leq 0.5$ \\
& & $($ sufficient conditions see App.B.2.4) \\
Section 4 & $>0($ sufficient conditions: & Depends on $\vartheta$ : \\
& $\vartheta<1$ and $\left.t_{a}+(1-2 \vartheta) c>0\right)$ & Always $\geq 0$ if $\vartheta \geq 0.5$. \\
& & Numeric testing reveals mostly positive \\
& & relationship; $K_{s}$ and $t_{a}$ sufficiently large will \\
& & as well ensure positive relationship
\end{tabular}

All results analytically derived, except for mentioned numeric testing.

An increase of $\vartheta$ intuitively leads to earlier replacement in sections 2 and 3 , in 4 it mostly leads to later replacement. This is natural in sections 2 and 3 . In section 4, the effect is due to the fact that early aging is already finished. An increase of $\vartheta$ thus reduces the magnitude of aging in this section and consequently lengthens replacement cycles. This might appear counterintuitive at first glance. This is due to following marginal consideration. It is optimal to replace relatively late because of relatively high reinvestment cost compared to penalties for premature replacement. Then relatively low additional risk of premature failure with increasing replacement age in section 4 becomes even lower because of increasing $\vartheta$. Trading off relatively high capital costs $K$ (which have to be high to be in section 4) against less important extra cost for premature replacement $s$ then leads to later optimal replacement. Finally, as well a higher level of $\vartheta$ itself will further increase the positive impact of an increase of $\vartheta$ on replacement age. The impact of $c$ on the solution depends majorly on the values for $\vartheta$, but a tendency of earlier replacement switching to later replacement with an increasing degree of uncertainty is observable. This switch may occur in section 3 , where, for $\vartheta=1$, the effect remains negative, but for lower $\vartheta$ (from a threshold $\vartheta$ onwards) the impact of increasing uncertainty may lead to later replacement (cf. Appendix B.2.4).

\section{Case B: Non-overlapping Failure Distributions}

In the non-overlapping case, aging will either happen early or late with no overlapping section of the respective functions for the different aging states ( $\mathrm{Cf}$. Figure 3, right hand side). This requires that $c m \geq 0.5$. In the following section, the range $\left[t_{a}-c ; t_{b}-c\right]$ will be referred to as 
section $2,\left[t_{b}-c ; t_{a}+c\right]$ as section 3 and $\left[t_{a}-c ; t_{b}+c\right]$ as section 4 . Regarding the functional forms of section 2 and 4 , distribution characteristics are very similar to their counterparts in the overlapping case A except for sections' boundaries. This entails identical functional forms for the calculation of optimal replacement ages $t_{j}^{* *}$ and an identical sensitivity of optimal replacement age to uncertainty parameters $\vartheta$ and $c$. The only section showing different functional forms differing from the overlapping distribution function is section 3 , as the following proposition shows.

Proposition 3 The unconditional failure rate of the mixture distribution function can be written as follows:

$$
f_{M}(t)= \begin{cases}0 & \text { for } t_{j}<t_{a}-c \\ \vartheta m & \text { for } t_{a}-c \leq t<t_{b}-c \\ 0 & \text { for } t_{b}-c \leq t<t_{a}+c \\ (1-\vartheta) m & \text { for } t_{a}+c \leq t<t_{b}+c \\ 0 & \text { for } t>t_{b}+c\end{cases}
$$

whereas the cumulative distribution function of failures is given by

$$
F_{M}(t)= \begin{cases}0 & \text { for } t<t_{a}-c \\ \vartheta m\left(t-\left(t_{a}-c\right)\right) & \text { for } t_{a}-c \leq t<t_{b}-c \\ \vartheta & \text { for } t_{b}-c \leq t<t_{a}+c \\ \vartheta+(1-\vartheta) m\left(t-\left(t_{a}+c\right)\right) & \text { for } t_{a}+c \leq t<t_{b}+c \\ 1 & \text { for } t_{b}+c \leq t\end{cases}
$$

and expected lifetime is

$$
G_{M}\left(t_{j}\right)= \begin{cases}t_{j} & \text { for } t_{j}<t_{a}-c \\ \vartheta\left(t_{j}-\frac{m}{2}\left(t_{j}-\left(t_{a}-c\right)\right)^{2}\right)+(1-\vartheta) t_{j} & \text { for } t_{a}-c \leq t_{j}<t_{b}-c \\ \vartheta\left(t_{b}-c-\frac{1}{2 m}\right)+(1-\vartheta) t_{j} & \text { for } t_{b}-c \leq t_{j}<t_{a}+c \\ \vartheta\left(t_{b}-c-\frac{1}{2 m}\right)+(1-\vartheta)\left(t_{j}-\frac{m}{2}\left(t_{j}-\left(t_{a}+c\right)\right)^{2}\right) & \text { for } t_{a}+c \leq t_{j}<t_{b}+c \\ \vartheta\left(t_{b}-c-\frac{1}{2 m}\right)+(1-\vartheta)\left(t_{b}+c-\frac{1}{2 m}\right) & \text { for } t_{b}+c \leq t_{j}\end{cases}
$$

Proofs of Proposition 3 Proofs analogously follow the proofs of Proposition 1. The proof for $F_{M}\left(t_{j}\right)$ in section 3 is obtained through a weighted sum of $F\left(t_{j}\right)$ in the early aging case by $\vartheta$, which has already taken its maximum value of 1 , and the late aging case by $(1-\vartheta)$, which still is equal to 0 in section 3 .

Consequently, aging occurs only in the second and the fourth section of the function. A major difference compared to the overlapping case is due to section 3, in which no failure occurs in the non-overlapping case. Consequently, the solutions of sections except section 3 are equal to the solutions in the previous application, the solution of section 3 being always $t_{a}+c$. However, the bounds of the solutions are different. This leads to the sole sensitivity of the optimal replacement age to $\vartheta$ and $c$, i.e. $t_{j}^{* *}$ increases proportionately to $c$ and is insensitive to $\vartheta$. 


\subsection{Optimal replacement when the period/speed of aging is uncertain}

Uncertainty about the slope of the cumulative failure distribution is represented via an additive modification of the slope $m$. In the case of slow aging, the unconditional failure rate is given by $m-z$ in the case of fast aging it is given by $m+z$. Obviously, $z$ has to be restricted to the case $z<m$ to avoid negative or zero aging in the first case.

Proposition 4 The unconditional failure distribution is given by:

$$
f_{M}(t)= \begin{cases}\vartheta(m+z)+(1-\vartheta)(m-z) & \text { for } t_{a}<t<t_{a}+\frac{1}{m+z} \\ (1-\vartheta)(m-z) & \text { for } t_{a}+\frac{1}{m+z}<t<t_{a}+\frac{1}{m-z} \\ 0 & \text { else }\end{cases}
$$

In the case of $\vartheta=0.5$ the function coincides in the first section with the function without uncertainty.

The cumulative failure distribution can then be derived.

$$
F_{M}(t)= \begin{cases}0 & \text { for } t<t_{a} \\ \left(t-t_{a}\right)(\vartheta(m+z)+(1-\vartheta)(m-z)) & \text { for } t_{a} \leq t<t_{a}+\frac{1}{m+z} \\ \vartheta+(1-\vartheta)(m-z)\left(t-t_{a}\right) & \text { for } t_{a}+\frac{1}{m+z} \leq t<t_{a}+\frac{1}{m-z} \\ 1 & \text { for } t \geq t_{a}+\frac{1}{m-z}\end{cases}
$$

The expected lifetime corresponds to:

$$
G_{M}\left(t_{j}\right)= \begin{cases}t_{j} & \text { for } t_{j}<t_{a} \\ t_{j}-\frac{m+2 z \vartheta-z}{2}\left(t_{j}-t_{a}\right)^{2} & \text { for } t_{a} \leq t_{j}<t_{a}+\frac{1}{m+z} \\ \vartheta\left(t_{a}+\frac{0,5}{m+z}\right)+(1-\vartheta)\left[t_{j}-\frac{m-z}{2}\left(t_{j}-t_{a}\right)^{2}\right] & \text { for } t_{a}+\frac{1}{m+z} \leq t_{j} \\ & \text { and } t_{j}<t_{a}+\frac{1}{m-z}\end{cases}
$$

Proofs Proposition 4 Cf. Appendix C.1.

Proposition 5 The optimal replacement strategy for the second section can be derived. The inner solution for section 2 is given by:

$$
t_{j}^{* *}=t_{a}+\frac{\sqrt{K_{s}^{2}+2 K_{s}-2(m+2 z \vartheta-z) t_{a}}-K_{s}}{m+2 z \vartheta-z}
$$

For $\vartheta=0.5, t_{j}^{* *}=t_{j}^{*}$ and there is no difference between the certain solution and the uncertain solution in section 2 . The inner optimum in section 3 corresponds to:

$$
t_{j}^{* *}=t_{a}+\frac{\sqrt{K_{s}^{2}-\vartheta^{2}+2 K_{s}+\frac{m+3 z+\vartheta(m-z)}{m+z} \vartheta-2(1-\vartheta)(m-z) t_{a}}-K_{s}-\vartheta}{(1-\vartheta)(m-z)}
$$




\section{Proofs Proposition 5 Cf. Appendix C.2.}

Section 1 and 4 are characterized by corner solutions corresponding to $t_{a}$ (section 1 ) and $t_{a}+1 /(m-z)$ (section 4). The probability of fast aging, $\vartheta$, and its impact, denoted $z$, define in which section an optimum will appear. If the probability of early aging is very low, then an optimal solution will always be found in section 3. Given very low uncertainty, which will result in $(m-z) /(m+z)$ close to 1 , the solution will be certainly found in section 2 because the section 3 is quasi not existing. A solution in section 2 is also very probable for low $K_{s}$ signifying high additional premature replacement costs.

The sensitivity of optimal replacement to uncertainty parameters is summarized in Table 2.

Table 2: Dependence of optimal replacement on $\vartheta$ and $z$

\begin{tabular}{lll}
\hline \hline Inner optimum & $\partial \boldsymbol{t}_{\boldsymbol{j}}^{* *} / \partial \boldsymbol{\vartheta}$ & $\partial \boldsymbol{t}_{\boldsymbol{j}}^{* *} / \partial \boldsymbol{z}$ \\
\hline \hline Section 2 & $<0$ & Depends on $\vartheta:$ \\
& & $<0$ if $\vartheta>0.5$ \\
& $=0$ if $\vartheta=0.5$ \\
Section 3 & $>0$ if $\vartheta<0.5$ \\
& $\begin{array}{l}\text { <0 (numeric testing results; exception: } \\
\text { unrealistic combination of very high } m t_{a} \\
\left.\text { combined with low } K_{s}\right)\end{array}$ & $>0$ \\
\hline
\end{tabular}

All results analytically derived, except for mentioned numeric testing.

In section 2, a higher $\vartheta$ will lead to earlier replacement. For symmetric $\vartheta$ no difference to the optimal replacement under certainty about aging parameters will occur. Then $t_{j}^{*}=t_{j}^{* *}$ and a variation of $z$ has no impact at all. The impact of the degree of uncertainty $z$ thus depends on $\vartheta$. For $\vartheta>0.5$, a higher probability of faster aging, uncertainty will lead to earlier replacement and vice versa. These effects are less obvious in section 3 . Here, numerical approaches reveal that an increase of $\vartheta$ also leads to earlier replacement. ${ }^{9}$ An increase of $z$ leads to later replacement. This mirrors to some extent the above result where increasing $c$ leads to later replacement in section 4 and partially in section 3 but to earlier replacement in section 2 and partially in section 3 .

\subsection{Computing the Value of Information}

The value of information indicates the benefits of relieving the uncertainty over the parameters of the failure rate distribution. For instance, if both possibilities (early and late aging) exist, knowing the actual failure rate distribution will allow informed choices of the optimal replacement time. The expected cost without information $(N-$ not informed) on the aging process is then given by:

$$
C^{N}=\frac{K+s F_{M}\left(t_{j}^{* * *}\right)}{G_{M}\left(t_{j}^{* *}\right)}
$$

With given information on the aging process, the distribution of the failure rate is known a priori $(I \text { - informed })_{F}$ Thus, the agent will use an optimal solution given unfavorable aging $t_{j}{ }^{U}$ or favorable aging $t_{j}{ }^{F}$ :

$$
C^{I}=\vartheta \frac{K+s F_{U}\left(t_{j}^{U}\right)}{G_{U}\left(t_{j}^{U}\right)}+(1-\vartheta) \frac{K+s F_{F}\left(t_{j}^{F}\right)}{G_{F}\left(t_{j}^{F}\right)}
$$

The value of information then corresponds to the difference between the costs in both states, namely 'uncertainty' or 'information' on the distribution of the failure rate:

$$
V=C^{N}-C^{I}
$$

\footnotetext{
${ }^{9}$ Cf. Appendix C.2.
} 


\section{Application}

This section discusses the impact of uncertainty in two specific application contexts. The first application presents the value of information generated by quality control of purchased components. In this case the example of VPE cables being subject to the water tree effect is considered. The willingness to pay for quality control depends on the benefit that will be achieved by optimizing maintenance. The second application discusses the benefit of an uncertain new technology, in our case given the example of new insulations for gas tubes.

\subsection{Willingness to Pay for Quality Control (water treeing)}

A first setting is the purchase of components which may be subject to quality control, allowing the detection of production flaws. The network operator will only carry out controls, if he knows that there may be a quality problem and that such problems may be detected by a quality control. If a component does not fulfill defined quality standards, the operator will not use it but instead give it back to the supplier or scrap it. Thereby he will be able to save future losses due to unforeseen, premature failure. A practical example is given by early VPE cables in the 1980 years. Due to water treeing effects, the cables' insulations deteriorated dramatically and whole charges of cables were replaced due to a high number of occurring failures.

A major question of interest is how the operator values the information about the quality of the component. This value $V$ corresponds perfectly to the sum he will be willing to pay for quality control. First, if no quality control is carried out the operator may receive a good component (with probability $l-\vartheta$ ), or a bad component with probability $\vartheta$. A bad component in this example corresponds to a VPE cable whose insulation deteriorates more rapidly due to water treeing. The cost is computed following (35).

In the informed state, he carries out quality controls and thus assures that components are always of good quality. In our case this means that the cable insulation will not suffer from any water tree impact. Consequently aging is slower, the slope of the cumulated failure distribution is given by $m-z$, and the operator will choose the corresponding informed strategy.

$$
C^{I}=\frac{K+s F_{m-z}\left(t_{j}^{m-z}\right)}{G_{m-z}\left(t_{j}^{m-z}\right)}
$$

Based on (37), the value of information corresponds to the cost difference between the uninformed and the informed state. The informed state is therefore supposed to be the state after the resolution of uncertainty about the speed of aging. The value of information depends on the probability $\vartheta$ of a component being subject to unfavorable aging and on the other parameters, like $z$. Therefore, a practical example based on published failure rate data from FGH (2006) will help deepening insights. In Figure 4 one can see that the first generation of VPE cables ('old') aged dramatically between an age of 20 and 40 years. 


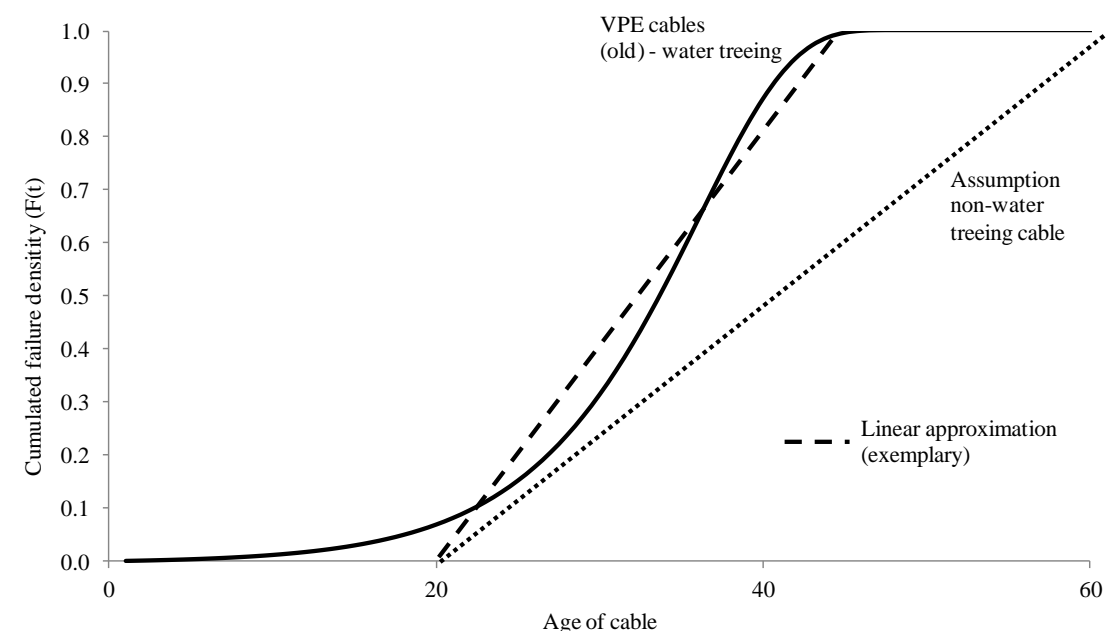

Figure 4: Cumulated failure rates for VPE cables (old), linear approximation and assumptions ${ }^{10}$

The figure illustrates that section 2, in which the two failure rates add up, ends with an age of 40 years, starts at $t_{a}=20$ years. Section 3 includes all ages from 41 up to 60 . For $s$, we assume different two scenarios: $20 \%$ of $K\left(K_{s}=5\right)$ and $50 \%$ of $K\left(K_{s}=2\right)$. $\vartheta$ can be interpreted as expected share of defective (e.g. water treeing) cables. Results for the value of information are given in the following table.

Table 3: Numerical results for section $2\left(m=0.0375, z=0.0125, t_{a}=20, K=100\right)$

\begin{tabular}{|c|c|c|c|c|c|c|c|c|c|c|c|c|}
\hline \multirow[b]{2}{*}{$\vartheta$} & \multicolumn{6}{|c|}{$K_{s}=5$} & \multicolumn{6}{|c|}{$K_{s}=2$} \\
\hline & $t_{j}^{* * *}$ & $t_{j}^{*}$ & $C^{I}$ & $C^{U}$ & $V$ & $V / C^{I}$ & $t_{j}^{* *}$ & $t_{j}^{*}$ & $C^{I}$ & $C^{U}$ & $V$ & $V / C^{I}$ \\
\hline 0.25 & 54 & 53 & 3.0 & 3.2 & 0.2 & $7 \%$ & 47 & 46 & 3.5 & 3.8 & 0.3 & $9 \%$ \\
\hline 0.50 & 54 & 53 & 3.0 & 3.4 & 0.4 & $15 \%$ & 35 & 46 & 3.5 & 4.2 & 0.6 & $18 \%$ \\
\hline 0.75 & 55 & 53 & 3.0 & 3.7 & 0.7 & $24 \%$ & 31 & 46 & 3.5 & 4.4 & 0.8 & $24 \%$ \\
\hline
\end{tabular}

The value of information describes the willingness to pay for the knowledge that reinvestment is with certainty performed using an asset of good quality. The value is important in all cases, corresponding to $7 \%$ up to $24 \%$ of the yearly maintenance costs in the informed state. $V$ increases with the probability of water treeing characteristics of the cable. For higher values of $K_{s}$, the replacement cycles is that long that it almost corresponds to the strategy used if defective cables were detected by quality control procedures. For lower values of $K_{s}$, a higher potential share of water-treeing cables $\vartheta$ leads to an important reduction of replacement cycles.

\subsection{Benefit of an Uncertain New Technology}

The next application compares two states: a costly state under parameter certainty and an uncertain but less costly state. This may for instance apply if a technology (e.g. a new type of insulations for steel tubes) is introduced that should lead to later aging, but its effectiveness cannot be guaranteed. Suppose that we have the cost function of an established asset class with aging speed $m$, aging starting at $t_{a}-c$.

$$
C^{I}=\frac{K+s F\left(t_{j}\right)}{G\left(t_{j}\right)}=\frac{K+s m\left(t_{j}^{*}-t_{a}+c\right)}{\int_{0}^{*}\left\{1-m\left(t-t_{a}+c\right)\right\}}
$$

\footnotetext{
${ }^{10}$ CDF of old VPE cables were derived from FGH (2006) and linearly approximated. The CDF regarding nonwater treeing cables represents an assumption.
} 
A new insulation is introduced that shall lead to later aging but no long term data is given to prove its impact. In the worst case, which occurs with probability $\vartheta$, it will have no effect at all. In the best case, aging will occur at $t_{a}+c$.

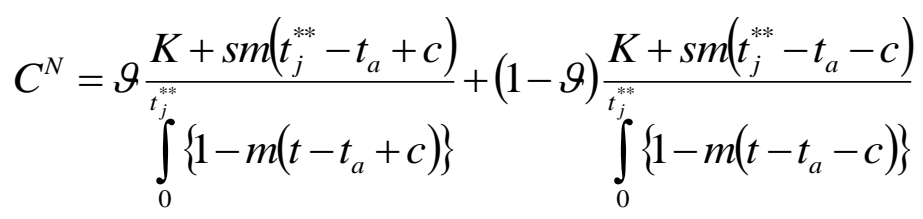

The uncertain cost advantage of the new technology $C^{\Delta}$ corresponds to the expected cost advantage of introducing the new technology, thus:

$$
\begin{aligned}
& C^{\Delta}=C^{I}-C^{N}= \\
& \vartheta\left[\frac{K+s m\left(t_{j}^{*}-t_{a}+c\right)}{\int_{0}^{* *}\left\{1-m\left(t-t_{a}+c\right)\right\}}-\frac{K+s m\left(t_{j}^{* * *}-t_{a}+c\right)}{\left.t_{j}^{* *}\left\{1-m\left(t-t_{a}+c\right)\right\}\right]} \int_{0}^{t_{j}^{*}}\right]\left[\frac{K+\operatorname{sm}\left(t_{j}^{*}-t_{a}+c\right)}{\int_{0}^{t_{j}^{*}}\left\{1-m\left(t-t_{a}+c\right)\right\}}-\frac{K+s m\left(t_{j}^{* *}-t_{a}-c\right)}{t_{0}^{* * *}\left\{1-m\left(t-t_{a}-c\right)\right\}}\right]
\end{aligned}
$$

Obviously, $C^{\Delta}$ increases with the probability $1-\vartheta$ that the technology will lead to later aging. We use an example of cumulated failure density for different types of insulations of steel tubes published in DVGW (1999), namely steel tubes with paper insulations and steel tubes with PE/fiber cement insulations. The normal distributions published are approximated by uniformly distributed unconditional failure rates (cf. Figure 5). We consider the case, that the aging characteristics of the new technology are uncertain. We assume the probability $\vartheta$ (unfavorable case), that the aging characteristics of the more recent insulation are not better than the characteristics of paper insulations.

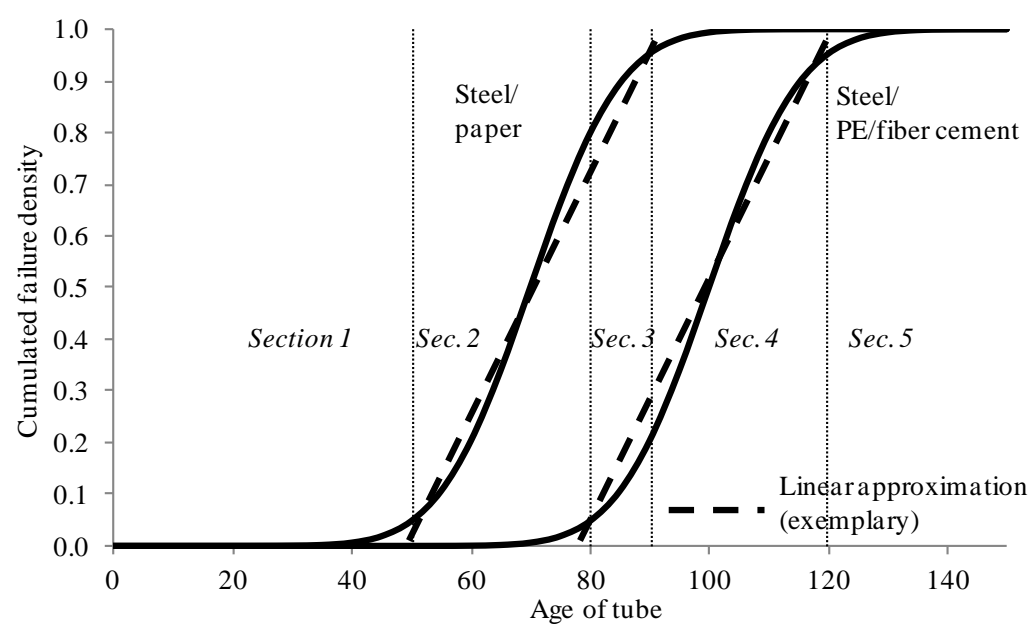

Figure 5: Cumulated failure rates for gas and linear approximation

The relevant distribution parameters to compute the value of information are $t_{a}=65, c=15$ and $m=0.025$. This corresponds to an overlapping case because in section 3 (ages between 80 and 90 years), both cumulated failure densities overlap. Again, extra costs of $20 \%$ and $50 \%$ for premature replacement are assumed. 
Table 4: Numerical results for section $2\left(m=0.025, c=15, t_{a}=65, K=100\right)$

\begin{tabular}{|c|c|c|c|c|c|c|c|c|c|c|c|c|}
\hline & $K_{s}=5$ & & & & & & $\boldsymbol{K}_{s}=$ & & & & & \\
\hline$\vartheta$ & $t_{j}^{* * *}$ & $t_{j}^{*}$ & $C^{I}$ & $C^{N}$ & $C^{4}$ & $C^{d} / C^{I}$ & $t_{j}^{* * *}$ & $t_{j}^{*}$ & $C^{I}$ & $C^{N}$ & $C^{\Delta}$ & $C^{\Delta} / C^{I}$ \\
\hline 0.25 & 101 & 78 & 1.7 & 1.3 & 0.4 & $22 \%$ & 80 & 64 & 1.9 & 1.4 & 0.5 & $25 \%$ \\
\hline 0.50 & 103 & 78 & 1.7 & 1.4 & 0.3 & $16 \%$ & 77 & 64 & 1.9 & 1.6 & 0.3 & $16 \%$ \\
\hline 0.75 & 82 & 78 & 1.7 & 1.6 & 0.1 & $6 \%$ & 75 & 64 & 1.8 & 1.8 & 0.0 & $1 \%$ \\
\hline
\end{tabular}

The intuitive result is obtained that the more the investor expects the favorable, old aging case to occur the more he is willing to pay for the knowledge enabling him to exclude the occurrence of the unfavorable aging case. For most cases, the cost difference is important. Only in one case (when the new insulation will not be better with a probability of $75 \%$ and premature replacement is costly), then the cost difference amount to only $1 \%$. For high $K_{s}$ implying low cost for premature replacement, the counterintuitive effect described in section 4.1.A is visible. Inner optimum solutions will mostly be within section 4 , and thus increase with $\vartheta$. For lower values of $K_{s}$ (the example on the right), optimal replacement decreases with the probability of unfavorable aging. The cost advantage due to the new technology is important, reducing costs by up to $25 \%$ in our example. Replacement cycles are extended by 10 to 26 years (compared to the 30 years difference between the failure rates).

\section{Conclusion}

This article analyzes the impact of uncertainty, concerning the beginning as well as the speed of the aging process, on the optimal replacement strategy. The unconditional failure rate was assumed to follow a uniform distribution, representing a plausible approximation of empirically observable aging functions and permitting to analytically derive an optimal replacement strategy. Considering uncertainty is achieved by mixing a favorable and an unfavorable failure pattern and analytically deriving the optimal strategy with an underlying mixed failure distribution.

First, we find that the characteristics of uncertainty have major impacts on the replacement decision. Higher degrees of uncertainty may lead to either earlier replacement or later replacement depending both on the optimal replacement age relative to the maximum asset age and on the probability the investor attributes to the occurrence of unfavorable aging cases. For earlier optimal replacement ages additional uncertainty, $c$, will induce shorter replacement cycles whereas the contrary is true for later optimal replacement ages. This stems from the tradeoff between reinvestment cost and costs for premature replacement: If latter are already relatively important additional uncertainty will enforce the effect towards early replacement. If on the other hand reinvestment cost exhibit a pressure to replace relatively late, additional uncertainty in tendency delays reinvestment because of even lower mixed failure probabilities. Similarly, the probability of occurrence of unfavorable aging cases, $\vartheta$, will reduce optimal replacement age for early replacement cases and increase it for late replacement cases. Second, a value of information as difference between the cost in an informed and an uninformed state was computed in the case of two applications. It is shown to be highly relevant and suggests a willingness to pay for the elimination of uncertainty of up to a fourth of realized costs.

As for possible future research, an area of interest would be to decrease the limitation by switching from a two-state-model to a multi-state or continuous model. 


\section{Appendix A: Example of linear approximation of Weibull-CDF}

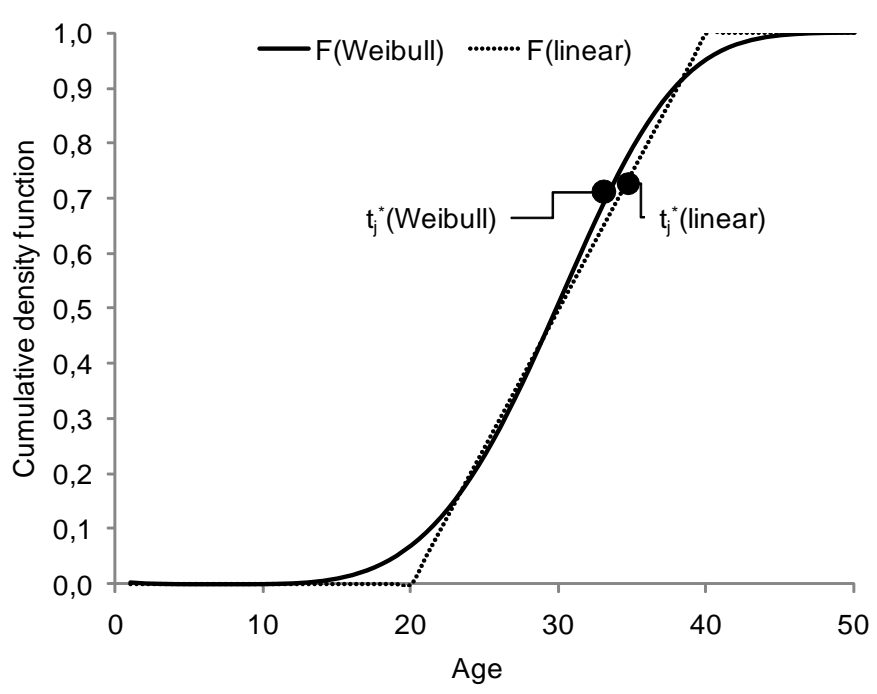

$$
\begin{aligned}
& \text { Weibull } \\
& F(t)= \begin{cases}0 & , \text { for } t \leq \gamma \\
1-e^{-\alpha(t-\gamma)^{\beta}} & , \text { for } t>\gamma\end{cases} \\
& \alpha=0,04 \quad \beta=4 \quad \gamma=7 \\
& F_{\text {lin }}(t)=\left\{\begin{array}{ll}
0 & , \text { for } t_{j}<t_{a} \\
m\left(t_{j}-t_{a}\right) & , \text { for } t_{a} \leq t_{j}<t_{b} \\
1 & , \text { for } t_{j} \geq t_{b}
\end{array}\right\} \\
& m=20 \quad t_{a}=0,05 \\
& \text { Comparision of Results (with } s=1, K=4 \text { ) } \\
& \begin{array}{llrr}
\text { tj } & \text { [years] } & 33 & 34 \\
\text { C+S } & \text { [annuity] } & 0,167 & 0,163 \\
\text { C+S } & \text { [Index] } & 1 & 0,98
\end{array}
\end{aligned}
$$

\section{Appendix B: Mixture of early and late aging - overlapping case}

\section{B.1 Calculus of $F_{M}(t)$ and $G_{M}\left(t_{j}\right)$}

\section{B.1.1 Calculus of $F_{M}(t)$}

Formally, the cumulative failure distribution function (cdf) is obtained through integration by parts over the support of $f_{M}(t): F_{M}(t)=\int_{0}^{t} f_{M}(\tau) d \tau$. As the cdf $F_{M}(t)$ is linear in $f_{M}(t)$ and the mixture distribution $f_{M}(t)$ is a linear combination of the unfavorable and favorable case, denoted $f_{E}(t)$ and $f_{L}(t)$ respectively. Correspondingly $F_{M}(t)$ can also be obtained as a weighted sum of the individual $F_{E}(t)$ and $F_{L}(t), F_{M}(t)=\vartheta F_{E}(t)+(1-\vartheta) F_{L}(t)$.

For the first and the last section $F_{M}(t)$ is simply 0 and 1 . In section 2 , the cdf is given by

$$
\begin{aligned}
F_{M}\left(t \mid t_{a}-c \leq t<t_{a}+c\right) & =\underbrace{t_{0}^{t_{a}-c} f_{M}\left(\tau \mid \tau<t_{a}-c\right) d \tau}_{=0}+\int_{t_{a}-c}^{t} f_{M}\left(\tau \mid t_{a}-c \leq \tau<t_{a}+c\right) d \tau=\int_{t_{a}-c}^{t} \vartheta m d \tau \\
& =\vartheta m\left(t-\left(t_{a}-c\right)\right) .
\end{aligned}
$$

In section 3, similarly

$$
\begin{aligned}
F_{M}\left(t \mid t_{a}+c \leq t<t_{b}-c\right) & =\vartheta m\left(\left(t_{a}+c\right)-\left(t_{a}-c\right)\right)+\int_{t_{a}+c}^{t} f_{M}\left(\tau \mid t_{a}+c \leq \tau<t_{b}-c\right) d \tau=m\left(t-\left(t_{a}+c\right)+2 \vartheta c\right) \\
& =\vartheta m\left(t-\left(t_{a}-c\right)\right)+(1-\vartheta) m\left(t-\left(t_{a}+c\right)\right) .
\end{aligned}
$$

For section 4,

$$
F_{M}\left(t \mid t_{b}-c \leq t<t_{b}+c\right)=\vartheta+(1-\vartheta) m\left(t-\left(t_{a}+c\right)\right) \text {. }
$$

Altogether, 


$$
F_{M}(t)= \begin{cases}0 & \text { for } t<t_{a}-c \\ \vartheta m\left(t-\left(t_{a}-c\right)\right) & \text { for } t_{a}-c \leq t<t_{a}+c \\ \vartheta m\left(t-\left(t_{a}-c\right)\right)+(1-\vartheta) m\left(t-\left(t_{a}+c\right)\right) & \text { for } t_{a}+c \leq t<t_{b}-c \\ \vartheta+(1-\vartheta) m\left(t-\left(t_{a}+c\right)\right) & \text { for } t_{b}-c \leq t<t_{b}+c \\ 1 & \text { for } t_{b}+c \leq t\end{cases}
$$

\section{B.1.2 Calculus of $G_{M}\left(t_{j}\right)$}

The expected utilization period is $G_{M}\left(t_{j}\right)=\int_{o}^{t_{j}}\left(1-F_{M}(\tau)\right) d \tau$. Similar to $F_{M}(t)$ the derivation of $G_{M}\left(t_{j}\right)$ is also possible by weighting $G_{E}\left(t_{j}\right)$ and $G_{L}\left(t_{j}\right), G_{M}\left(t_{j}\right)=\vartheta G_{E}\left(t_{j}\right)+(1-\vartheta) G_{L}\left(t_{j}\right)$.

With $t_{j}$ being part of section $1, G_{M}\left(t_{j}\right)$ then is simply $t_{j}$.

In section 2,

$$
\begin{aligned}
G_{M}\left(t_{j} \mid t_{a}-c \leq t_{j}<t_{a}+c\right) & =\int_{o}^{t_{a}-c}\left(1-F_{M}\left(\tau \mid \tau<t_{a}-c\right)\right) d \tau+\int_{t_{a}-c}^{t_{j}}\left(1-F_{M}\left(\tau \mid t_{a}-c \leq \tau<t_{a}+c\right)\right) d \tau \\
& =\int_{0}^{t_{a}-c} 1 d \tau+\int_{t_{a}-c}^{t_{j}}\left(1-\vartheta m\left(\tau-\left(t_{a}-c\right)\right)\right) d \tau=t_{j}-\frac{\vartheta m}{2}\left(t_{j}-\left(t_{a}-c\right)\right)^{2} \\
& =\vartheta\left(t_{j}-\frac{m}{2}\left(t_{j}-\left(t_{a}-c\right)\right)^{2}\right)+(1-\vartheta) t_{j} .
\end{aligned}
$$

In section $3, G_{M}\left(t_{j}\right)$ is given by

$$
\begin{aligned}
G_{M}\left(t_{j} \mid t_{a}+c \leq t_{j}<t_{b}-c\right) & =G_{M}\left(t_{a}+c\right)+\int_{t_{a}+c}^{t_{j}}\left(1-F_{M}\left(\tau \mid t_{a}+c \leq \tau<t_{b}-c\right)\right) d \tau \\
& =t_{j}-\frac{m}{2}\left[\left(t_{j}-t_{a}\right)^{2}+c^{2}+2 c(1-2 \vartheta)\left(t_{a}-t_{j}\right)\right] \\
& =\vartheta\left(t_{j}-\frac{m}{2}\left(t_{j}-\left(t_{a}-c\right)\right)^{2}\right)+(1-\vartheta)\left(t_{j}-\frac{m}{2}\left(t_{j}-\left(t_{a}+c\right)\right)^{2}\right) .
\end{aligned}
$$

For section $4, G_{M}\left(t_{j}\right)$ is obtained according to following formula.

$$
\begin{aligned}
G_{M}\left(t_{j} \mid t_{b}-c \leq t_{j}<t_{b}+c\right) & =G_{M}\left(t_{b}-c\right)+\int_{t_{b}-c}^{t_{j}}\left(1-F_{M}\left(\tau \mid t_{b}-c \leq \tau<t_{b}+c\right)\right) d \tau \\
& =\vartheta\left(t_{b}-c-\frac{1}{2 m}\right)+(1-\vartheta)\left(t_{j}-\frac{m}{2}\left(t_{j}-\left(t_{a}+c\right)\right)^{2}\right) .
\end{aligned}
$$

Section 5 is similarly given by

$$
\begin{aligned}
G_{M}\left(t_{j} \mid t_{b}+c<t_{j}\right) & =G_{M}\left(t_{b}+c\right)+\int_{t_{b}+c}^{t_{j}}\left(1-F_{M}\left(\tau \mid t_{b}+c<\tau\right)\right) d \tau=t_{b}-\frac{1}{2 m}+c(1-2 \vartheta) \\
& =\vartheta\left(t_{b}-c-\frac{1}{2 m}\right)+(1-\vartheta)\left(t_{b}+c-\frac{1}{2 m}\right) .
\end{aligned}
$$

Overall, 


$$
G_{M}\left(t_{j}\right)= \begin{cases}t_{j} & \text { for } t_{j}<t_{a}-c \\ \vartheta\left(t_{j}-\frac{m}{2}\left(t_{j}-\left(t_{a}-c\right)\right)^{2}\right)+(1-\vartheta) t_{j} & \text { for } t_{a}-c \leq t_{j}<t_{a}+c \\ \vartheta\left(t_{j}-\frac{m}{2}\left(t_{j}-\left(t_{a}-c\right)\right)^{2}\right)+(1-\vartheta)\left(t_{j}-\frac{m}{2}\left(t_{j}-\left(t_{a}+c\right)\right)^{2}\right) & \text { for } t_{a}+c \leq t_{j}<t_{b}-c \\ \vartheta\left(t_{b}-c-\frac{1}{2 m}\right)+(1-\vartheta)\left(t_{j}-\frac{m}{2}\left(t_{j}-\left(t_{a}+c\right)\right)^{2}\right) & \text { for } t_{b}-c \leq t_{j}<t_{b}+c \\ \vartheta\left(t_{b}-c-\frac{1}{2 m}\right)+(1-\vartheta)\left(t_{b}+c-\frac{1}{2 m}\right) & \text { for } t_{b}+c \leq t_{j}\end{cases}
$$

\section{B.2 Optimality conditions}

The cost function is given by equation (9)

$$
C\left(t_{j}\right)=\frac{K+s F\left(t_{j}\right)}{G\left(t_{j}\right)}
$$

with the resulting optimality condition (11): $r\left(t_{j}\right) G\left(t_{j}\right)-F\left(t_{j}\right)-K_{s}=0$.

\section{B.2.1 Inner local optimum in section 2}

$$
\begin{aligned}
& r_{M}\left(t_{j}\right) G_{M}\left(t_{j}\right)-F_{M}\left(t_{j}\right)-K_{s}=0 \\
& \Leftrightarrow \frac{\vartheta m}{1-\vartheta m\left(t_{j}-t_{a}+c\right)}\left[t_{j}-\frac{\vartheta m}{2}\left(t_{j}-t_{a}+c\right)^{2}\right]-\vartheta m\left(t_{j}-t_{a}+c\right)-K_{s}=0 \\
& \Leftrightarrow \frac{\vartheta^{2} m}{2} t_{j}^{2}+\frac{\vartheta^{2} m}{2} t_{a}^{2}+\frac{\vartheta^{2} m}{2} c^{2}-\vartheta^{2} m t_{j} t_{a}+\vartheta^{2} m t_{j} c-\vartheta^{2} m t_{a} c+\vartheta t_{a}-\vartheta c=\frac{K_{s}}{m}-K_{s} \vartheta t_{j}+K_{s} \vartheta t_{a}-K_{s} \vartheta c \\
& \Leftrightarrow t_{j}{ }^{2}\left(\frac{\vartheta m}{2}\right)+t_{j}\left(-\vartheta m t_{a}+\vartheta m c+K_{s}\right)+\left(t_{a}-c+\frac{1}{2} \vartheta m t_{a}^{2}-\vartheta m t_{a} c+\frac{1}{2} \vartheta m c^{2}-\frac{K_{s}}{\vartheta m}-K_{s} t_{a}+K_{s} c\right)=0
\end{aligned}
$$

The quadratic equation yields as feasible solution:

$t_{j}=t_{a}-c$

$$
+\frac{\sqrt{\left(-\vartheta_{m} t_{a}+\vartheta_{m}+K_{s}\right)^{2}-4\left(\frac{\vartheta_{m}}{2}\right)\left(t_{a}-c+\frac{1}{2} \vartheta_{m t_{a}}{ }^{2}-\vartheta_{m} t_{a} c+\frac{1}{2} \vartheta_{m} c^{2}-\frac{K_{s}}{\vartheta_{m}}-K_{s} t_{a}+K_{s} c\right)}-K_{s}}{\vartheta_{m}}
$$

The term under the square root may be simplified further to obtain $K_{s}^{2}+2 K_{s}-2 \vartheta m t_{a}+2 \vartheta m c$

This leads to the following result

$$
t_{j}^{* *}=t_{a}-c+\frac{\sqrt{K_{s}^{2}+2 K_{s}-2 \vartheta m\left(t_{a}-c\right)}-K_{s}}{\vartheta m} .
$$

\section{B.2.2 Properties of an inner local optimum in section 2}

In this section, the sensitivity of the optimal solution with respect to $\vartheta$ and $c$ is analyzed. The sensitivity with respect to $\vartheta$ is:

$\frac{\partial t_{j}^{* *}}{\partial \vartheta}<0$ 
This follows directly from the fact that the square root is decreasing in $\vartheta$ whereas the denominator is increasing in $\vartheta$.

The impact of $c$ on the optimal replacement time is given by:

$$
\frac{\partial t_{j}^{* *}}{\partial c}=-1+\frac{1}{\sqrt{K_{s}^{2}+2 K_{s}-2 \vartheta m\left(t_{a}-c\right)}}
$$

An inner optimum will only occur for $t_{j}^{* *}$ larger then $t_{a}-c$ (cf. equation (A1)). This requires the square root to be larger than $K_{s}$ according to equation (A1). Thus a sufficient condition for the derivative with respect to $c$ to be positive is $K_{s}>1$. This condition is not restrictive in practice, given that $K_{s}$ corresponds to the ratio of normal replacement costs to the additional cost (or penalty) for early replacement. Thus in any case where the penalty does not exceed the normal replacement costs, the derivative with respect to $c$ will be negative.

\section{B.2.3 Inner local optimum in section 3}

The locally optimal solution for section 3 is computed from

$$
\begin{aligned}
& r_{M}\left(t_{j}\right) G_{M}\left(t_{j}\right)-F_{M}\left(t_{j}\right)-K_{s}=0 \\
& \Leftrightarrow \frac{m}{1-m\left(t_{j}-t_{a}-c+2 \vartheta c\right)}\left[t_{j}-\frac{m}{2}\left[\left(t_{j}-t_{a}\right)^{2}+c^{2}+2 c(1-2 \vartheta)\left(t_{a}-t_{j}\right)\right]\right]-m\left(t_{j}-t_{a}-c+2 \vartheta c\right)-K_{s}=0 \\
& \Leftrightarrow \frac{m}{2} t_{j}^{2}+t_{j}\left(-m t_{a}+2 \vartheta m c-m c+K_{s}\right) \\
& \quad+\left(t_{a}+c-2 \vartheta c+\frac{m}{2} t_{a}{ }^{2}+\frac{m}{2} c^{2}+m t_{a} c-2 \vartheta m t_{a} c+4 \vartheta^{2} m c^{2}-4 \vartheta m c^{2}-\frac{K_{s}}{m}-K_{s} t_{a}-K_{s} c+2 \vartheta K_{s} c\right)=0 .
\end{aligned}
$$

This leads to:

$$
\begin{aligned}
& t_{j}=t_{a}+c(1-2 \vartheta)-\frac{K_{s}}{m}+ \\
& \frac{1}{m} \sqrt{\left(-m t_{a}+2 \vartheta m c-m c+K_{s}\right)^{2}-m\left(\begin{array}{l}
2 t_{a}+2 c-4 \vartheta c+m t_{a}^{2}+m c^{2}+2 m t_{a} c-4 \vartheta m t_{a} c+\ldots \\
\ldots+8 \vartheta^{2} m c^{2}-8 \vartheta m c^{2}-\frac{2 K_{s}}{m}-2 K_{s} t_{a}-2 K_{s} c+4 \vartheta K_{s} c
\end{array}\right)}
\end{aligned}
$$

Simplifying the term under the square root gives the following result

$$
t_{j}^{* *}=t_{a}+(1-2 \vartheta) c+\frac{\sqrt{K_{s}^{2}+2 K_{s}-2 m t_{a}+4 m^{2}\left(\vartheta-\vartheta^{2}\right) c^{2}-2 m(1-2 \vartheta) c}-K_{s}}{m}
$$

\section{B.2.4 Properties of an inner local optimum in section 3}

In this section, the sensitivity of the optimal solution with respect to $\vartheta$ and $c$ is analyzed. For $\vartheta$, the various terms containing $\vartheta$ react differently: 


$$
t_{j}^{* *}=t_{a}+\overbrace{(1-2 \vartheta) c}^{\frac{\partial(\cdot)}{\partial \vartheta}<0}+\frac{\sqrt{K_{s}^{2}+2 K_{s}-2 m t_{a}+\overbrace{4 m^{2}\left(\vartheta-\vartheta^{2}\right) c^{2}}^{\frac{\partial(\cdot)}{\partial \vartheta}>0 \text {,if } \vartheta<0.5}+\overbrace{2 m(2 \vartheta-1) c}^{\frac{\partial(\cdot)}{\partial \vartheta}>0}-K_{s}}}{m} .
$$

Thus the sign of the derivative is not obvious at first sight.

Writing $k=K_{s}^{2}+2 K_{s}-2 m t_{a}$, the derivative is given by:

$$
\begin{aligned}
\frac{\partial t_{j}^{* *}}{\partial \vartheta} & =-2 c+\frac{4 c^{2} m^{2}(1-2 \vartheta)+4 c m}{2 m \sqrt{k+4 c^{2} m^{2}\left(\vartheta-\vartheta^{2}\right)+2 c m(2 \vartheta-1)}} \\
& =2 c\left(-1+\frac{c m(1-2 \vartheta)+1}{\sqrt{k+4 c^{2} m^{2}\left(\vartheta-\vartheta^{2}\right)+2 c m(2 \vartheta-1)}}\right)
\end{aligned}
$$

As in the previous case, the solution for $t_{j}^{* *}$ from equation (A2) is only an interior solution, if the square root fulfils certain conditions. Since section 3 by definition starts from $t=t_{a}+c$, the following condition has to be fulfilled for an interior solution:

$$
\sqrt{k+4\left(\vartheta-\vartheta^{2}\right) c^{2} m^{2}+2(2 \vartheta-1) c m}>K_{s}+2 \vartheta c m
$$
)

Under this condition an upper limit for the derivative is

$$
\frac{\partial t_{j}^{* *}}{\partial \vartheta}<2 c\left(-1+\frac{c m(1-2 \vartheta)+1}{K_{s}+2 \vartheta c m}\right)
$$

The worst case is then $\vartheta=0$ and $\mathrm{cm}=1 / 2$ (larger values of $\mathrm{cm}$ correspond rather to the nonoverlapping case). Still a value of $K_{s}$ above $3 / 2$ is then sufficient to ensure negativity of the derivative. This is typically not a strong restriction on $K_{s}$, especially since the restrictions for an interior solution mostly put tighter restrictions on $K_{s}$

With respect to changes in $c$, simple inspection indicates different reactions:

$$
t_{j}^{* *}=t_{a}+\frac{\overbrace{c m(1-2 \vartheta)}^{\frac{\partial(\cdot)}{\partial c}<0, \text { if } \vartheta>0.5}+\sqrt{K_{s}^{2}+2 K_{s}-2 m t_{a}+\overbrace{4 c^{2} m^{2}\left(\vartheta-\vartheta^{2}\right)}^{\frac{\partial(\cdot)}{\partial c}>0}+\overbrace{2 c m(2 \vartheta-1)}^{\frac{\partial(\cdot)}{\partial c}<0, \text { if } \vartheta<0.5}}-K_{s}}{m} .
$$

The derivative is then:

$$
\frac{\partial t_{j}^{* *}}{\partial c}=(1-2 \vartheta)+\frac{4 c m\left(\vartheta-\vartheta^{2}\right)-(1-2 \vartheta)}{\sqrt{K_{s}^{2}+2 K_{s}-2 m t_{a}+4 c^{2} m^{2}\left(\vartheta-\vartheta^{2}\right)+2 c m(2 \vartheta-1)}}
$$

Thus the derivative with respect to $c$ is strongly dependent on the values of $\vartheta$. The first term in the derivative thereby dominates in almost all cases compared to the third one, given the previously obtained condition for the square root (cf. equation (A3)) and typical values of $K_{s}$. The maximum value of the second term in the derivative is 0.5 for $\vartheta=1 / 2$ and $\mathrm{cm}=1 / 2$. Thus this term is also of less importance and has only a decisive impact on the sign of the derivative for $\vartheta$ close to 0.5 . Inspection of configurations for extreme values for $\vartheta$ confirms this perception. In order to obtain a positive derivative $\partial t_{j}^{* *} / \partial c>0$, the following conditions have to be met. 


$$
\begin{array}{lll}
\vartheta=0: & \sqrt{k-2 c m}>1 \Leftrightarrow & k>2 c m+1 \\
\vartheta=0.5: & & 0<c m \\
\vartheta=1: & & \sqrt{k+2 c m}<1 \Leftrightarrow
\end{array}
$$

For $\vartheta=1$ the inequality is hardly ever fulfilled given typical values for $K_{s}$. When $\mathrm{cm}$ takes its maximum value the inequality does not hold and even for the other extreme value, $\mathrm{cm}=0, \mathrm{k}$ would have to be smaller than one. Consequently increasing uncertainty as measured through $c$ will lead to shorter replacement cycles for $\vartheta=1$ in most cases. Contrarily, for $\vartheta=0.5$ the inequality is always true, thus larger $c$ will always lead to longer replacement cycles independent of parameterizations. For the lowest possible value of $\vartheta, \vartheta=0$, the inequality is also fulfilled for typical values of $K_{s}$, hence increasing uncertainty $c$ usually leads to increasing optimal replacement age. Nevertheless, in the unusual case that $k \leq 2 \mathrm{~cm}+1$ shorter replacement cycles due to uncertainty might as well occur.

\section{B.2.5 Inner local optimum in section 4}

The locally optimal solution for section 4 is computed from

$$
\begin{aligned}
& r_{M}\left(t_{j}\right) G_{M}\left(t_{j}\right)-F_{M}\left(t_{j}\right)-K_{s}=0 \\
& \Leftrightarrow \frac{(1-\vartheta) m}{1-\vartheta-(1-\vartheta) m\left(t_{j}-t_{a}-c\right)}\left[\vartheta\left(t_{b}-c-\frac{1}{2 m}\right)+(1-\vartheta)\left[t_{j}-\frac{m}{2}\left(t_{j}-t_{a}-c\right)^{2}\right]\right] \\
& -\vartheta-(1-\vartheta) m\left(t_{j}-t_{a}-c\right)-K_{s}=0 \\
& \Leftrightarrow(1-\vartheta) m t_{j}^{2}+2\left(-m t_{a}-c m+\vartheta m t_{a}+\vartheta c m+\vartheta+K_{s}\right) t_{j} \\
& +\left[\vartheta\left(t_{a}+t_{b}\right)-2 \vartheta c+m t_{a}{ }^{2}+c^{2} m+2 c m t_{a}-\vartheta m t_{a}{ }^{2}-\vartheta m c^{2}\right. \\
& \left.\quad-2 \vartheta c m t_{a}-2 \frac{\vartheta}{m}+2 t_{a}+2 c-4 \vartheta t_{a}-4 \vartheta c-2 \frac{K_{s}}{m}-2 K_{s} t_{a}-2 c K_{s}\right]=0 \\
& \Leftrightarrow(1-\vartheta) m t_{j}^{2}+2\left(-m t_{a}-c m+\vartheta m t_{a}+\vartheta c m+\vartheta+K_{s}\right) t_{j} \\
& +\left[\vartheta\left(t_{a}+t_{b}\right)-2 \vartheta c+m t_{a}^{2}+c^{2} m+2 c m t_{a}-\vartheta m t_{a}{ }^{2}-\vartheta m c^{2}\right. \\
& \left.\quad-2 \vartheta c m t_{a}-2 \vartheta\left(t_{b}-t_{a}\right)+2 t_{a}+2 c-4 \vartheta t_{a}-4 \vartheta c-2 K_{s} t_{b}-2 c K_{s}\right]=0 \\
& \Leftrightarrow(1-\vartheta) m t_{j}{ }^{2}+2\left(-m t_{a}-c m+\vartheta m t_{a}+\vartheta c m+\vartheta+K_{s}\right) t_{j} \\
& +\left[-\vartheta\left(t_{b}+t_{a}\right)-2 \vartheta c+m t_{a}{ }^{2}+c^{2} m+2 c m t_{a}-\vartheta m t_{a}{ }^{2}-\vartheta m c^{2}\right. \\
& \left.\quad-2 \vartheta c m t_{a}+2 t_{a}+2 c-4 \vartheta c-2 K_{s} t_{b}-2 c K_{s}\right]=0
\end{aligned}
$$

Again a quadratic equation thus has to be resolved. For the term under the square root one obtains:

$$
\begin{aligned}
& 4\left(\vartheta-m t_{a}-c m+\vartheta m t_{a}+\vartheta c m+K_{s}\right)^{2} \\
& \begin{array}{r}
4(1-\vartheta) m\left[-\vartheta\left(t_{a}+t_{b}\right)-2 \vartheta c+m t_{a}{ }^{2}+c^{2} m+2 c m t_{a}-\vartheta m t_{a}{ }^{2}-\vartheta m c^{2}\right. \\
\left.\quad-2 \vartheta c m t_{a}+2 t_{a}+2 c-4 \vartheta t_{a}-4 \vartheta c-2 K_{s} t_{b}-2 c K_{s}\right]
\end{array} \\
& =4\left[K_{s}{ }^{2}+2 K_{s}-2 m t_{a}-\vartheta^{2}+2 \vartheta+(3-\vartheta) \vartheta m t_{a}-(1-\vartheta) \vartheta m t_{b}+2 c m\left(3 \vartheta-1-2 \vartheta^{2}\right)\right] \\
& =4\left[K_{s}{ }^{2}+2 K_{s}+\vartheta-2(1-\vartheta) m\left(t_{a}+(1-2 \vartheta) c\right)\right]
\end{aligned}
$$


The optimal replacement age is then given by

$$
\begin{aligned}
& t_{j}^{* *}=\frac{-2\left(\vartheta-(1-\vartheta) m\left(t_{a}+c\right)+K_{s}\right) \pm 2 \sqrt{K_{s}^{2}+2 K_{s}+\vartheta-2(1-\vartheta) m\left(t_{a}+(1-2 \vartheta) c\right)}}{2(1-\vartheta) m} \\
& t_{j}^{* *}=t_{a}+c+\frac{\sqrt{K_{s}^{2}+2 K_{s}+\vartheta-2(1-\vartheta) m\left(t_{a}+(1-2 \vartheta) c\right.}-\vartheta-K_{s}}{(1-\vartheta) m} \\
& t_{j}^{* *}=t_{b}+c+\frac{\sqrt{K_{s}^{2}+2 K_{s}+\vartheta-2(1-\vartheta) m\left(t_{a}+(1-2 \vartheta) c\right)}-1-K_{s}}{(1-\vartheta) m}
\end{aligned}
$$

\section{B.2.6 Properties of an inner local optimum in section 4}

In this section, the sensitivity of the optimal solution with respect to $\vartheta$ and $c$ is considered. The derivative with respect to $\vartheta$ is:

$$
\frac{\partial t_{j}^{* *}}{\partial \vartheta}=+\frac{1}{(1-\vartheta)^{2} m}\left(\frac{(1-\vartheta)\left(1+\vartheta+2 m\left(t_{a}+(1-2 \vartheta) c\right)+4(1-\vartheta) m c\right)}{2 \sqrt{K_{s}^{2}+2 K_{s}+\vartheta-2(1-\vartheta) m\left(t_{a}+(1-2 \vartheta) c\right)}}+\sqrt{K_{s}^{2}+2 K_{s}+\vartheta-2(1-\vartheta) m\left(t_{a}+(1-2 \vartheta) c\right)}\right)
$$

As long as $\vartheta<1$ and $t_{a}+(1-2 \vartheta) c>0$, all terms are positive and thus the derivative is positive.

For the derivative with respect to $c$ one obtains:

$$
\frac{\partial t_{j}^{* *}}{\partial c}=+1-\frac{1-2 \vartheta}{\sqrt{K_{s}^{2}+2 K_{s}+\vartheta-2(1-\vartheta) m\left(t_{a}+(1-2 \vartheta) c\right)}}
$$

Here the result is less obvious. A sufficient condition for a positive derivative obviously is given by $\vartheta>1 / 2$. Moreover $K_{s}$ and $t_{a}$ sufficiently large will ensure a positive derivative.

\section{Appendix C: Mixture of fast and slow aging}

\section{C.1 Calculus for distribution functions}

\section{C.1.1 Density function $f\left(t_{j}\right)$}

Again, the unconditional failure distribution function is given by the mixture distribution of the respective fast and slow aging functions. For section 2

$$
f_{M}\left(t_{j}\right)=\vartheta(m+z)+(1-\vartheta)(m-z)=m+2 z \vartheta-z
$$

and for section 3

$f_{M}\left(t_{j}\right)=(1-\vartheta)(m-z)$.

\section{C.1.2 Cumulative distribution function $F\left(t_{j}\right)$}

The CDF of failures is given by

$$
\begin{aligned}
F_{M}\left(t_{j}\right) & =\vartheta(m+z)\left(t_{j}-t_{a}\right)+(1-\vartheta)(m-z)\left(t_{j}-t_{a}\right) \\
& =\left(t_{j}-t_{a}\right)[\vartheta(m+z)+(1-\vartheta)(m-z)]
\end{aligned}
$$


for section 2 and for section 3 by

$F_{M}\left(t_{j}\right)=\vartheta+(1-\vartheta)(m-z)\left(t_{j}-t_{a}\right)$.

As $F_{M}$ is the integral over $f_{M}$ it is continuous.

\section{C.1.3 Average life time $G\left(t_{j}\right)$}

The mixture of slow and fast aging subsamples leads to $G$ given by

$G_{M}\left(t_{j}\right)=\vartheta\left(t_{j}-\frac{m+z}{2}\left(t_{j}-t_{a}\right)^{2}\right)+(1-\vartheta)\left(t_{j}-\frac{m-z}{2}\left(t_{j}-t_{a}\right)^{2}\right)=t_{j}-\frac{m+2 z \vartheta-z}{2}\left(t_{j}-t_{a}\right)^{2}$

for section 2 and for section 3 by

$$
\begin{aligned}
& G_{M}\left(t_{j}\right)=\vartheta\left(t_{a}+\frac{1}{m+z}-\frac{m+z}{2}\left(t_{a}+\frac{1}{m+z}-t_{a}\right)^{2}\right)+(1-\vartheta)\left(t_{j}-\frac{m-z}{2}\left(t_{j}-t_{a}\right)^{2}\right) \\
& =\vartheta\left(t_{a}+\frac{1}{2(m+z)}\right)+(1-\vartheta)\left(t_{j}-\frac{m-z}{2}\left(t_{j}-t_{a}\right)^{2}\right)
\end{aligned}
$$

As $G_{M}$ is the integral over $F_{M}$ it is continuous.

\section{C.2 Optimal Replacement Age}

As before the cost function is given by equation (9)

$$
C\left(t_{j}\right)=\frac{K+s F\left(t_{j}\right)}{G\left(t_{j}\right)}
$$

with the resulting optimality condition $r\left(t_{j}\right) G\left(t_{j}\right)-F\left(t_{j}\right)-K_{s}=0$.

\section{C.2.1 Inner local optimum in section 2}

For an inner local optimum the first-order condition yields:

$$
\begin{aligned}
& r_{M}\left(t_{j}\right) G_{M}\left(t_{j}\right)-F_{M}\left(t_{j}\right)-K_{s}=0 \\
& \Leftrightarrow K_{s}\left(1-F\left(t_{j}\right)\right)=f\left(t_{j}\right) G\left(t_{j}\right)-F\left(t_{j}\right)\left(1-F\left(t_{j}\right)\right) \\
& \Leftrightarrow K_{s}\left(1-\left(t_{j}-t_{a}\right) m_{z, \vartheta}\right)=m_{z, \vartheta}\left[t_{j}-\frac{m_{z, \vartheta}}{2}\left(t_{j}-t_{a}\right)^{2}\right]-\left(t_{j}-t_{a}\right) m_{z, \vartheta}\left(1-\left(t-t_{a}\right) m_{z, \vartheta}\right) \\
& \Leftrightarrow 0=\left(\frac{m_{z, \vartheta}}{2}\right) t_{j}{ }^{2}+\left(-m_{z, \vartheta} t_{a}+K_{s}\right) t_{j}+\left(\frac{m_{z, \vartheta}}{2} t_{a}{ }^{2}+t_{a}-\frac{K_{s}}{m_{z, \vartheta}}-K_{s} t_{a}\right)
\end{aligned}
$$

Employing the formula for the solution of quadratic equations gives as single feasible solution 


$$
\begin{aligned}
& \Rightarrow t_{j}^{* * *}=\frac{-\left(-m_{z, \vartheta} t_{a}+K_{s}\right)+\sqrt{\left(-m_{z, \vartheta} t_{a}+K_{s}\right)^{2}-4\left(\frac{m_{z, \vartheta}}{2}\right)\left(\frac{m_{z, \vartheta}}{2} t_{a}^{2}+t_{a}-\frac{K_{s}}{m_{z, \vartheta}}-K_{s} t_{a}\right)}}{2\left(\frac{m_{z, \vartheta}}{2}\right)} \\
& =t_{a}+\frac{\sqrt{K_{s}^{2}+2 K_{s}-2 m_{z, \vartheta} t_{a}}-K_{s}}{m_{z, \vartheta}}=t_{a}+\frac{\sqrt{K_{s}^{2}+2 K_{s}-2(m+2 z \vartheta-z) t_{a}}-K_{s}}{m+2 z \vartheta-z},
\end{aligned}
$$

This solution corresponds to the solution under certainty about aging parameters, when $\vartheta=$ 0.5 is assumed:

$$
t_{j}^{* *} \stackrel{\vartheta=0.5}{=} t_{a}+\frac{\sqrt{K_{s}^{2}+2 K_{s}-2 m t_{a}}-K_{s}}{m} .
$$

\section{C.2.2 Properties of inner local optimum in section 2}

Since the numerator in the expression for $t_{j}^{* *}$ is decreasing and the denominator increasing in $\vartheta$, the derivative with respect to $\vartheta$ obviously fullfils:

$$
\frac{\partial t_{j}^{* *}}{\partial \vartheta}<0
$$

For the derivative with respect to the speed of aging $z$ a similar reasoning may be applied after slight reformulation of the expression for $t_{j}^{* * *}$ :

$$
t_{j}^{* *}=t_{a}+\frac{\sqrt{K_{s}^{2}+2 K_{s}-2(m+(2 \vartheta-1) z) t_{a}}-K_{s}}{m+(2 \vartheta-1) z},
$$

Then the sign of the derivative is clearly dependent on the value of $\vartheta$ :

$$
\frac{\partial t_{j}^{* *}}{\partial z} \begin{cases}<0 & \text { for } \vartheta>\frac{1}{2} \\ =0 & \text { for } \vartheta=\frac{1}{2} \\ >0 & \text { for } \vartheta<\frac{1}{2} .\end{cases}
$$

\section{C.2.3 Inner local optimum in section 3}

From the first order condition we get:

$$
\begin{aligned}
& r_{M}\left(t_{j}\right) G_{M}\left(t_{j}\right)-F_{M}\left(t_{j}\right)-K_{s}=0 \\
& \Leftrightarrow K_{s}\left(1-\vartheta-(1-\vartheta)(m-z)\left(t_{j}-t_{a}\right)\right)=(1-\vartheta)(m-z)\left[\vartheta\left[t_{a}+0.5 \frac{1}{m+z}\right]+(1-\vartheta)\left[t_{j}-\frac{m-z}{2}\left(t_{j}-t_{a}\right)^{2}\right]\right] \\
& -\left(\vartheta+(1-\vartheta)(m-z)\left(t_{j}-t_{a}\right)\left(1-\vartheta-(1-\vartheta)(m-z) t_{j}\left(t-t_{a}\right)\right)\right. \\
& \Leftrightarrow 0=t_{j}^{2}\left[\frac{(m-z)(1-\vartheta)}{2}\right]+t_{j}\left[-(1-\vartheta)(m-z) t_{a}+K_{s}+\vartheta\right] \\
& +\left[\frac{(m-z)(1-\vartheta)}{2} t_{a}^{2}+0.5 \frac{\vartheta}{m+z}-\frac{\vartheta}{m-z}-\vartheta t_{a}+t_{a}-\frac{K_{s}}{m-z}-K_{s} t_{a}\right]
\end{aligned}
$$

Application of the solution formula for quadratic equations gives 


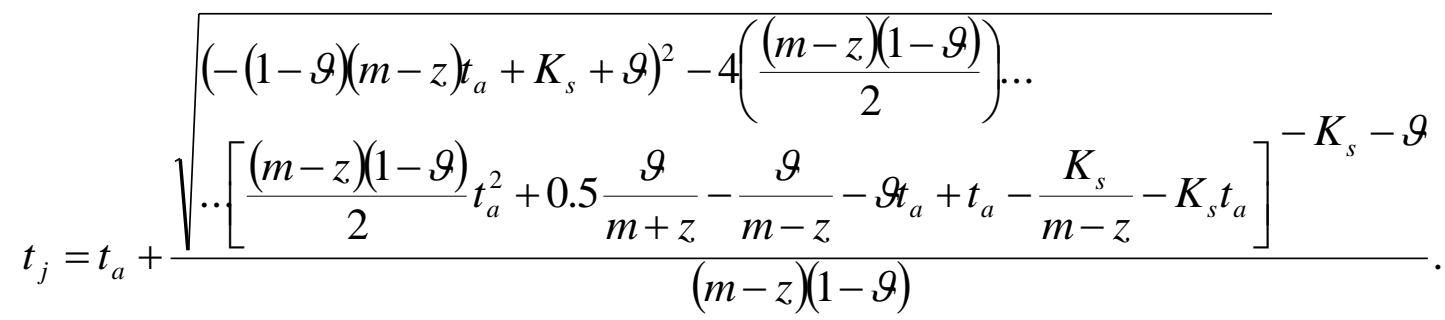

The expression under the square root may be simplified:

$$
\begin{aligned}
& \left(-(1-\vartheta)(m-z) t_{a}+K_{s}+\vartheta\right)^{2} \\
& -4\left(\frac{(m-z)(1-\vartheta)}{2}\right)\left[\frac{(m-z)(1-\vartheta)}{2} t_{a}^{2}+0.5 \frac{\vartheta}{m+z}-\frac{\vartheta}{m-z}-\vartheta t_{a}+t_{a}-\frac{K_{s}}{m-z}-K_{s} t_{a}\right] \\
& =K_{s}{ }^{2}-\vartheta^{2}+2 K_{s}+\frac{m+3 z+\vartheta(m-z)}{m+z} \vartheta-2(1-\vartheta)(m-z) t_{a}
\end{aligned}
$$

This leads to the optimal replacement age

$$
t_{j}^{* *}=t_{a}+\frac{\sqrt{K_{s}^{2}-\vartheta^{2}+2 K_{s}+\frac{m+3 z+\vartheta(m-z)}{m+z} \vartheta-2(1-\vartheta)(m-z) t_{a}}-K_{s}-\vartheta}{(m-z)(1-\vartheta)} .
$$

\section{C.2.4 Properties of an inner local optimum in section 3}

Given the different terms in the expression for the optimal replacement age and their dependency on $\vartheta$, no clear result can be easily derived for the derivative with respect to $\vartheta$.

For the derivative with respect to $z$, we find:

$$
\frac{\partial t_{j}^{* *}}{\partial z}>0
$$

because

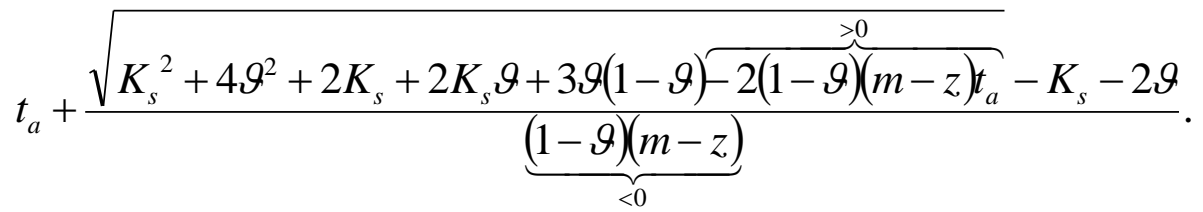




\section{List of Symbols}

$c \quad$ Uncertainty related to the starting point of aging

$C \quad$ Total equipment cost (objective function)

$C^{I} \quad$ Total equipment cost (objective function) under parameter certainty ('informed')

$C^{N} \quad$ Total equipment cost (objective function) under parameter uncertainty ('uninformed')

$C^{\Delta} \quad$ Cost difference between informed and uninformed state

$f \quad$ Probability density function of unconditional failure

$F \quad$ Probability distribution function of unconditional failure

$G \quad$ Mean lifetime of asset class

$H \quad$ Probability distribution function of age (age structure of assets)

$j \quad$ Assets class

$m \quad$ Slope of probability distribution function

$\vartheta \quad$ Probability of early or fast aging

$R_{\text {corr }} \quad$ Probability of premature/corrective replacement

$R_{\text {prev }} \quad$ Probability of normal/preventive replacement

$r \quad$ Conditional failure probability (failure rate)

$S \quad$ Total penalty cost

$s \quad$ Additional cost for breakdown

$t_{a} \quad$ Starting point of the aging period

$t_{b} \quad$ End of the aging period

$t_{j_{*}} \quad$ Planned utilization period

$t_{j_{* *}} \quad$ Optimal replacement strategy under parameter certainty

$t_{j}^{* *} \quad$ Planned utilization period under parameter uncertainty

$V \quad$ Value of information

$z \quad$ Uncertainty related to the speed of aging

$Z \quad$ Replacement cost of equipment

\section{References}

Barlow, R. E.; Hunter, L. (1960): Optimal preventive maintenance policies; Operations Research (VOL. 8); pp.80-90

Barlow, R. E.; Proschan, F. (1964): Comparison of replacement policies and renewal theory implications; Annals of Mathematical Statistics (VOL. 35); pp.577-589

Bassin, W. M. (1973): A Bayesian Optimal Overhaul Interval Model for the Weibull Restoration Process Case; Journal of the American Statistical Association (VOL. 68); pp.575-578

Dogramaci, A.; Fraiman, N. M. (2004): Replacement Decisions with Maintenance Under Uncertainty: An Imbedded Optimal Control Model; Operations Research (VOL. 52); pp.785-794

DVGW (1999): Technische Regel Arbeitsblatt G 401 - Entscheidungshilfen für die Rehabilitation von Gasverteilungsnetzen, September1999

FGH (2006): Technischer Bericht 299 - Asset-Management von Verteilungsnetzen - Komponentenverhalten und Analyse des Kostenrisikos; Forschungsgemeinschaft für Elektrische Anlagen und Stromwirtschaft e. V.

Finkelstein, M. (2008): Failure Rate Modelling for Reliability and Risk; Springer Series in Reliability Engineering (); pp.

Fox, B. (1966): Age Replacement with Discounting; Operations Research (VOL. 14); pp.533-537

Huang, G.Q.; Lau, S. K.; Mak, K. L. (2003): The impacts of sharing production information on supply chain dynamics: a review of the literature; International Journal of Production Research (VOL. 41); pp.14831517

Juang, M.-G.; Anderson, G. (2004): A Bayesian method on adaptive preventive maintenance problem; European Journal of Operational Research (VOL. 155); pp.455-473

Kamien, M. I.; Schwarz, N. L. (1971): Optimal maintenance and sale age for a machine subject to failure; Management Science (VOL. 17); pp.427-449

Ketzenberg, M. (2009): The value of information in a capacitated closed loop supply chain; European Journal of Operational Research (VOL. 198); pp.491-503 
Lee, C. (2008): A Bayesian approach to determine the value of information in the newsboy problem; International Journal of Production Economics (VOL. 112); pp.391-402

Mazzuchi, T. A.; Soyer, R. (1996): A Bayesian perspective on some replacement strategies; Reliability Engineering \& Systems Safety (VOL. 51); pp.295-303

Sathe, P. T.; Hancock, W. M. (1973): A Bayesian approach to the scheduling of preventive maintenance; AIIE Transactions (VOL. 5); pp.172-179

Valdez-Flores, C.; Feldman, R. C. (1989): A Survey of Preventive Maintenance Models for Stochastically Deteriorating Single-Unit Systems; Naval Research Logistics (VOL. 36); pp.419-446

Wang, H. (2002): A survey of maintenance policies of deteriorating systems; European Journal of Operational Research (VOL. 139); pp.469-489

Wang, L.; Chu, J.; Mao, W. (2009): A condition-based replacement and spare provisioning policy for deteriorating systems with uncertain deterioration to failure; European Journal of Operational Research (VOL. 194); pp.184-205

Weber, C.; Schober, D.; Schaeffler, S. (2010): Optimal Replacement Strategies in Network Infrastructures under Quality Penalties; Zeitschrift für Betriebswirtschaft (VOL. 80); pp.639-655

Yatsenko, Y.; Hritonenko, N. (2008): Properties of optimal service life under technological change; International Journal of Production Economics (VOL. 114); pp.130-138 